\title{
Alteration of Structure, Electronic, and Vibrational Properties of amorphous GeTe by Selenium substitution: an Experimentally Constrained Density Functional Study
}

\author{
M. Micoulaut ${ }^{1}$, A. Piarristeguy ${ }^{2}$, O. Masson $^{3}$, R. Escalier ${ }^{2}$, H. Flores-Ruiz ${ }^{4}$, A. Pradel ${ }^{2}$ \\ ${ }^{1}$ Sorbonne Université, Laboratoire de Physique Théorique de la Matière Condensée, \\ CNRS UMR 7600, 4 Place Jussieu, 75252 Paris Cedex 05, France \\ 2 ICGM, Université de Montpellier, CNRS, ENSCM, Montpellier, France. \\ ${ }^{3}$ Institut de Recherche sur les Céramiques, UMR 7315 CNRS - Université de Limoges \\ Centre Européen de la Céramique, 12 rue Atlantis 87068 Limoges Cedex, France \\ ${ }^{4}$ Departamento de Ciencias Naturales y Exactas, CUValles, Universidad de Guadalajara, \\ Carr. Guadalajara-Ameca km 45.5, 46600, Ameca, Jalisco. México
}

(Dated: October 12, 2021)

\begin{abstract}
The structural, vibrational and electronic properties of several compositions of amorphous Ge-Se-Te are studied from a combination of X-ray diffraction and density functional-based molecular dynamics. Different structural properties are considered such as structure factors, pair distribution functions, angular distributions, coordination numbers and neighbor distributions. We compare results with experimental findings and a satisfying agreement is found for the structure functions in real and reciprocal spaces. The short range order is found to be more complex than in related binaries that result in mixed geometries $(\simeq 65-75 \%$ tetrahedral, and remaining defect octahedral) for a dominant four-fold $\mathrm{Ge}(80 \%)$. The chalcogen atoms are dominantly 2-fold, the former having furthermore an important fraction of 3-fold coordinated atoms (30-40\%). The obtained model structures indicate that $\mathrm{Ge}-\mathrm{Ge}, \mathrm{Ge}-\mathrm{Se}$, and $\mathrm{Ge}-\mathrm{Te}$ bonds dominate with small fractions of Te-Te bonds remaining from the base system GeTe. The investigation of electronic properties indicates that the addition of Se atoms will lead to Te-related bands that are much more localized so that Ge-Te-Se can be regarded as having an increased covalent character with respect to GeTe.
\end{abstract}

\section{INTRODUCTION}

Telluride based crystalline and amorphous materials can be used for a rather wide range of promising applications such as thermoelectrics ${ }^{1,2}$, infrared waveguides ${ }^{3,4}$ and flash memory devices ${ }^{5,6}$ using the phase change mechanism. For the latter, the material undergoes a short and reversible transition between the amorphous and crystalline states which display a strong optical or electrical contrast that is central for data storage. Typical identified compounds are found on the lie-line $\mathrm{GeTe}-\mathrm{Sb}_{2} \mathrm{Te}_{3}$. The prototypal phase change material (PCM) $\mathrm{Ge}_{2} \mathrm{Sb}_{2} \mathrm{Te}_{5}$ (GST) has some of these characteristics but appears to be not promising enough for phase-change random access memory applications because of low crystallization temperature, a poor data retention ability and a low resistance contrast ${ }^{7}$. Targeted additives have been suggested to improve the performances such as $\mathrm{Ag}^{8}$ or $\mathrm{Bi}^{9}$.

Selenium also appears to be attractive due its smaller size and it is more covalent than Te. Recent studies ${ }^{10-12}$ have emphasized the promising role of Se in the enhancement of phase change properties. It is therefore tempting to investigate the effect of Se addition into another typical PCM, that is, GeTe, because of its improved crystallization temperature, data retention and resistance contrast capabilities with respect to $\mathrm{GST}^{13}$. In this respect, the addition of Se into GeTe has been considered recently ${ }^{14}$ and results on thin films indicate that with Se addition the contrast in electrical resistivity could be increased up to a factor of 100 between the amorphous and crystalline material states ${ }^{15}$, other properties being continuously controlled by Se content such as resistance, bandgap, transition temperature and threshold voltage. Here, we remind that the c-GeTe occurs in two forms: a trigonal $\mathrm{R}_{3 m}$ symmetry that is stable at ambient conditions ${ }^{16}$ up to $50-50.5$ at. $\%$ Te and a cubic rocksalt structure stable at high temperatures. The nature of these phases as well as their stability has been examined in Ref. 17. Conversely, c-GeSe occurs in a orthorhombic $\mathrm{P}_{n m a}$ symmetry up to the melting point at $667^{\circ} \mathbf{C}^{18,19}$.

The effect of Se addition on Ge-Te amorphous networks has been only considered in the Te-rich domain using as base glass $\mathrm{GeTe}_{4}$ or similar compositions for infrared optical fiber applications ${ }^{20-23}$. Recent density function theory (DFT), Raman spectroscopy and Nuclear Magnetic Resonance (NMR) investigations have been also performed on Se$\mathrm{GeTe}_{4}$ glasses $^{26,27}$. The effect of Se on the base glass appears to be different as the substitution of Se into this kind of chalcogen-rich base network also alters Te-chains which are completely absent in $\mathrm{GeTe}^{28}$. The mechanism of substitution and the involved structural modification must be, thus, very different. Except few experimental studies ${ }^{15,24,25}$, we are not aware of any other study on the Te/Se substitution in the base amorphous GeTe material. This is the purpose of the present contribution.

Here we present a combined experimental and computational study on amorphous $\mathrm{GeTe}_{1-x} \mathrm{Se}_{x}$ systems for a different degree of Se/Te substitution $x$. Results from X-ray diffraction experiments are combined with density functional based molecular dynamics simulations. This permits a complete description of the structural, vibrational and electronic properties, while also providing a neat account for the effect of Se substitution into the prototypal phase change material GeTe. Structural properties are illustrated by the structure factors, radial distribution functions, coordination numbers, nature of the neighbors and angular distributions. The main outcome is the fact that such amorphous systems do not contain at all chalcogen-chalcogen homopolar bonds (Te-Te, $\mathrm{Te}-\mathrm{Se}, \mathrm{Se}-\mathrm{Se}$ ), the only homopolar bonds present being Ge- 
Ge. Regarding the local structure of Germanium, we also show that the networks evolve from a mixture of tetrahedral (T) and octahedral $(\mathrm{O})$ Ge to a dominant tetrahedral network in $\mathrm{GeSe}$ that contains however large bond-bending motions driven by the stress-release of highly cross-linked amorphous networks. Regarding chemical bonding, we demonstrate that the addition of Se increases the covalent character of Te-based bonds as the localization of corresponding 4s-orbitals is substantially increased for the considered ternary compositions $\mathrm{GeSe}_{0.5} \mathrm{Te}_{0.5}$ and $\mathrm{GeSe}_{0.75} \mathrm{Te}_{0.25}$.

\section{METHODS}

\section{A. Film elaboration}

$\mathrm{GeTe}_{1-x} \mathrm{Se}_{x}(\mathrm{x}=0,0.50,0.75,1.0)$ thin films (thicknesses $6 \mu \mathrm{m}$ ) were deposited by thermal co-evaporation of the pure elements (Ge pieces, Te pieces and Se granules from ChemPur, each one with a purity of 99.999\%) using Plassys MEB 500 device equipped with two current induced heating sources and an electron beam evaporator. The three sources were placed in a configuration that allowed the deposition of films with uniform composition and thickness over a surface of about $4 \mathrm{~cm}$ in diameter. The two current induced heated sources were used to evaporate Selenium and Tellurium, whereas the electron beam was used to evaporate Germanium. Se and Te were placed in two carbon crucibles inserted in Molybdenum nacelles covered with a perforated Molybdenum foil, in order to ensure a stable evaporation rate. Germanium was placed in the electron beam using a Copper crucible. The microscope slides used as substrates were cleaned with alcohol and dried with dry air. Before the deposition, the chamber was evacuated down to approximately $10^{-5} \mathrm{~Pa}$. During the deposition process, the substrate holder was rotating at $8 \mathrm{rpm}$ and heated at $90^{\circ} \mathrm{C}$ in order to improve the adhesion with the deposited layer. The evaporation rate and thickness for each element were automatically controlled with pre-calibrated quartz crystal monitors. A typical film deposition rate of $420 \mathrm{~nm} / \mathrm{min}$ was applied. Let us note that no further annealing treatment was carried out prior to proceeding to the film characterization.

\section{B. X-ray diffraction}

The experimental structure factors $S(k)$ and atomic pair distribution functions $g(r)$ of the samples were obtained by X-ray total scattering. Measurements were performed at room temperature with a dedicated laboratory setup based on a Bruker D8 advance diffractometer $(\lambda=0.559422 \AA)$ equipped with a silver sealed tube and a rapid LynxEye XE-T detector. This setup was modified in order to maximize collected intensities, to minimize spurious signal from the empty environment and to obtain a good counting statistics up to a large scattering vector length of $21.8 \AA^{-1}$. For each sample, a small amount of powder was placed in a thin-walled $(0.01 \mathrm{~mm})$ borosilicate glass capillary of about $0.3 \mathrm{~mm}$ in diameter. Once sealed, the

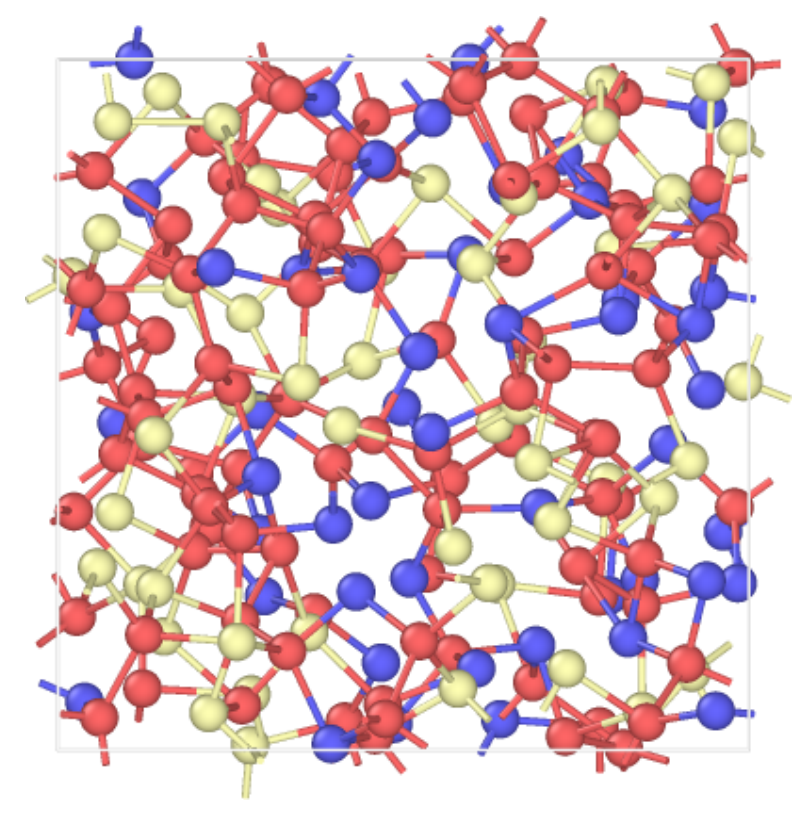

Figure 1: A typical snapshot of amorphous $\mathrm{GeSe}_{0.50} \mathrm{Te}_{0.50}$. Ge, $\mathrm{Se}$ and $\mathrm{Te}$ atoms are represented by red, blue and yellow spheres, respectively. Bonds are defined using a uniform cutoff of $3.1 \AA$.

capillary was mounted on a goniometric head and adjusted such that its axis coincides with the goniometer axis of the diffractometer. The data acquisition consisted of several scans in the $0.4-152^{\circ}, 50-152^{\circ}$ and $100-152^{\circ}$ ranges with a step size of $0.01^{\circ}$. The scans were subsequently merged, leading to a total equivalent acquisition time of about 120 hours per sample. The raw data were corrected, normalized and Fourier transformed using a homemade software ${ }^{41}$ in order to obtain the atomic pair distribution function of the sample, $g(r)$. The corrections included capillary, empty environment and Compton scatterings, fluorescence, absorption and polarization effects.

\section{RESULTS}

\section{A. Molecular dynamics}

We performed a series of first principles molecular dynamics simulations ${ }^{30}$ using a canonical (NVT) ensemble on four atomic $\mathrm{GeTe}_{1-x} \mathrm{Se}_{x}$ systems containing $N=200$ atoms with different compositions $\left(x=0^{29}, 0.50,0.75\right.$ and 1.0) with the number of $\mathrm{Ge}$, Te and Se atoms fulfilling the desired stoichiometry (Figure 1). A periodically repeated cubic cell was used, whose size changes according to the number density of the glasses ${ }^{24}$ (Table I). In order to check for pressure sensitivity, different additional simulations were performed for the particular $x=0.50$ system, and indicated that a pressure change from - 4.0 GPa up to 6.0 GPa led to a density variation of $\pm 20 \%$, i.e. we found $\rho_{0}=0.0263 \AA^{-3}$ for - 4.0 GPa, and 0.048 $\AA^{-3}$ for 6.0 GPa. At the experimental system density, the calculated pressure was found to 
be $\simeq-2.0 \mathrm{GPa}$ at $300 \mathrm{~K}$. Such negative values are known to result from the incorporation of dispersion forces ${ }^{31,32}$ which improve the structure and chemical bond lengths with respect to experiments in Tellurides but induce a slightly tensile character. We used density functional theory with a Grimme correction (DFT-D $2^{33}$ ) in combination with plane wave basis sets. The electronic scheme used a PBE functional ${ }^{34}$ within a generalized gradient approximation (GGA) for the exchange correlation energy. Previous investigations on the related binary GeTe have shown that this electronic scheme improves substantially the description of both short and intermediate-range in the liquid and amorphous state $^{29,35,36}$. The electronic structure of the liquids and glasses was described within DFT and evolved self-consistently during the motion with valence electrons being treated explicitly, in conjunction with norm-conserving pseudopotentials to account for core-valence interactions. The wave functions were expanded at the $\Gamma$-point of the supercell and the energy cutoff was set at $20 \mathrm{Ry}$. Starting configurations were taken from binary $\mathrm{GeTe}$ liquids $^{29}$ and Se atoms were randomly inserted in order to meet the desired stoichiometry.

Loss of the memory of the initial configurations has been achieved through preliminary runs at $2000 \mathrm{~K}$ over $30 \mathrm{ps}$ with a time step of $\Delta t=0.36 \mathrm{fs}$ and a fictitious mass of 2000 a.u., prior to equilibration at $1500 \mathrm{~K}, 1200 \mathrm{~K}, 900 \mathrm{~K}$ and $600 \mathrm{~K}$, each at $30-60 \mathrm{ps}$, and finally $300 \mathrm{~K}$ for $80 \mathrm{ps}$ (Figure 2a). These cooling steps were performed in a sequential fashion, e.g. the equilibration at $1200 \mathrm{~K}$ started from the last configuration (positions/velocities) obtained at $1500 \mathrm{~K}$, and so on. For each composition, the quenching procedure was repeated three times by selecting at $900 \mathrm{~K}$ independent configurations of the equilibrated liquids. By averaging over three independent trajectories in the glassy state which describe possible quenched structures of the potential energy landscape, one increases the statistical accuracy of the structural model as previously noticed for other systems ${ }^{37,38}$.

The addition of Se into the base GeTe leads to an overall stabilization of the system as the calculated Kohn-Sham energies $E_{K S}$ decrease with the addition of Se content. During the quenching of the liquids, $E_{K S}$ decrease with decreasing $T$ as it does during the experimental glass transition that can be also reproduced from simulation 39,40 . Figure 2a highlights such salient phenomenology for a given cooling rate in $\mathrm{GeSe}_{0.50} \mathrm{Te}_{0.50}$, and the small curvature in the region 500$1000 \mathrm{~K}$ might indicate the presence of a possible fictive temperature. It should be noted that for each target temperature, the systems is rather well equilibrated as shown in the inset which represents $E_{K S}$ with simulation time for different $T$. Figure $2 \mathrm{~b}$ now represents $E_{K S}$ for the four samples as a function of temperature. It is seen that the addition leads to a systematic decrease of $E_{K S}$ with increasing Se content.

\section{B. Reciprocal space properties}

We first represent in Fig. 3a the calculated and measured X-ray structure factor. Note that $S(k)$ has been calculated using an X-ray weighted sum of partial correlations $S_{n m}(k)$ in
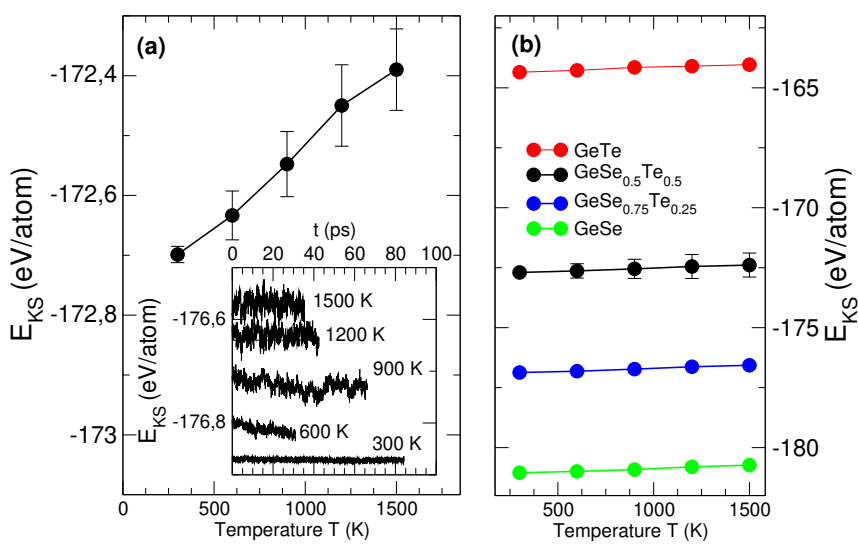

Figure 2: (a) Evolution of the Kohn-Sham energy $E_{K S}$ as a function of temperature $T$ for $\mathrm{GeSe}_{0.50} \mathrm{Te}_{0.50}$. The inset displays the time evolution of $E_{K S}$ over the trajectory. (b) Evolution of the Kohn-Sham energy $E_{K S}$ for the four systems of interest.

Fourier space :

$$
S(k)=\langle f\rangle^{-2} \sum_{n, m} c_{n} c_{m} f_{n} f_{m} S_{n m}(k)
$$

with :

$$
\langle f\rangle=\sum_{n} c_{n} f_{n}=f_{G e}+x f_{S e}+(1-x) f_{T e}
$$

where the $f_{n}$ and $c_{n}$ represent the atomic form factors taken as $f_{n}=Z_{n}\left(f_{G e}=32, f_{S e}=34, f_{T e}=52\right)$ and species concentration, respectively. The partial correlations have been evaluated from a Fourier transform of the partial pair correlation functions $g_{n m}(r)$ :

$$
S_{n m}(k)=1+\rho_{0} \int 4 \pi r^{2}\left[g_{n m}(r)-1\right] \frac{\sin (k r)}{k r} d r
$$

where $\rho_{0}$ is the system number density ${ }^{24}$ (Table I). Note that for select compositions, we have also used in parallel both equs. (3) and (4) (see below) :

$$
S_{n m}(k)=\frac{1}{N}\left\langle\sum_{n} \sum_{m} e^{-i \mathbf{k} .\left(\mathbf{R}_{n}-\mathbf{R}_{m}\right)}\right\rangle
$$

For all compositions, the comparison between the calculated $S(k)$ and XRD measured total structure factor appears to be very good as all peaks are nearly reproduced in position, amplitude and width. We notice however that the increase of Se content leads to a progressive overestimation of the first principal peak (PP) at $k_{1}$, although the other parts of the diffraction pattern are entirely reproduced up to $14 \AA^{-1}$ for amorphous GeSe. Of special importance is the reproduction of the two principal peaks (PP) for the other compositions found at $k_{1} \simeq 1.92-2.25 \AA^{-1}$ and $k_{2} \simeq 3.37-3.54 \AA^{-1}$, and the near absence of a first sharp diffraction peak that is barely visible in the experimental spectra (see however at $1.5 \AA^{-1}$ ). The addition of Se into the base GeTe leads to a slightly shifted higher $k$ values in principal peaks (PP) positions of 
Table I: Experimental (first line per composition) and calculated properties (second line in bracketts) of the different amorphous Ge-Se-Te systems : system number density, measured positions $k_{1}$ and $k_{2}$ of the first two principal peaks of the total structure factors $S(k)$, order parameter $S=S\left(k_{2}\right) / S\left(k_{1}\right)$, first- and second-neighbor peak positions $r_{1}$ and $r_{2}$ of the total pair correlation function $g(r)$, minimum $r_{\text {min }}$ of the pair correlation function $g(r)$, coordination number $N_{t o t}$ using the minimum $r_{\min }$ of the $g(r)$. Note that a cutoff of $2.2 \AA$ has been used for the evaluation of the experimental $N_{t o t}$ in order to avoid the spurious effects of short-range oscillations.

\begin{tabular}{|c|c|c|c|c|c|c|c|c|}
\hline System & $\rho_{0}\left(\AA^{-3}\right)^{24}$ & $k_{1}\left(\AA^{-1}\right)$ & $k_{2}\left(\AA^{-1}\right)$ & $S$ & $r_{1}(\AA)$ & $r_{2}(\AA)$ & $r_{\text {min }}$ & $N_{t o t}$ \\
\hline GeTe & 0.0328 & $\begin{array}{l}1.92(1) \\
(2.02)\end{array}$ & $\begin{array}{c}3.39(0) \\
(3.37)\end{array}$ & $\begin{array}{c}0.98(7) \\
(0.97)\end{array}$ & $\begin{array}{c}2.59(6) \\
(2.63)\end{array}$ & $\begin{array}{c}4.15(6) \\
(4.00)\end{array}$ & $\begin{array}{c}3.16(0) \\
(3.16)\end{array}$ & $\begin{array}{c}2.70(1) \\
(2.76)\end{array}$ \\
\hline $\mathbf{G e S e}_{0.50} \mathbf{T e}_{0.50}$ & 0.0347 & $\begin{array}{l}1.92(5) \\
(2.04)\end{array}$ & $\begin{array}{c}3.44(3) \\
(3.44)\end{array}$ & $\begin{array}{c}1.10(6) \\
(1.05)\end{array}$ & $\begin{array}{c}2.48(0) \\
(2.54)\end{array}$ & $\begin{array}{c}3.91(8) \\
(4.02)\end{array}$ & $\begin{array}{c}3.09(4) \\
(3.04)\end{array}$ & $\begin{array}{c}2.81(4) \\
(2.98)\end{array}$ \\
\hline $\mathbf{G e S e}_{0.75} \mathbf{T e}_{0.25}$ & 0.0371 & $\begin{array}{l}1.94(2) \\
(2.12)\end{array}$ & $\begin{array}{c}3.49(2) \\
(3.49)\end{array}$ & $\begin{array}{c}1.08(9) \\
(1.09)\end{array}$ & $\begin{array}{c}2.42(2) \\
(2.43)\end{array}$ & $\begin{array}{c}3.93(0) \\
(3.87)\end{array}$ & $\begin{array}{c}3.06(1) \\
(3.02)\end{array}$ & $\begin{array}{c}2.99(2) \\
(3.18)\end{array}$ \\
\hline GeSe & 0.0381 & $\begin{array}{c}2.02(6) \\
(2.25)\end{array}$ & $\begin{array}{c}3.53(2) \\
(3.54)\end{array}$ & $\begin{array}{c}1.22(6) \\
(1.16)\end{array}$ & $\begin{array}{c}2.40(0) \\
(2.45)\end{array}$ & $\begin{array}{c}3.86(8) \\
(3.86)\end{array}$ & $\begin{array}{c}2.92(5) \\
(2.94)\end{array}$ & $\begin{array}{c}3.01(4) \\
(3.09)\end{array}$ \\
\hline
\end{tabular}

experimental and calculated $S(k)$, especially for the second peak position $k_{2}$. Even the behavior up to $k \simeq 15 \AA^{-1}$ is reproduced and this becomes obvious once the interference function $I(k)=k[S(k)-1]$ is represented (Figure 3b) as $I(k)$ blows up the oscillations at large momentum transfer. The agreement is, thus, an indication that the short-range order of the Ge-Se-Te networks is correctly reproduced. In addition, a Gaussian decomposition in Fourier space of $S(k)$ has shown that features beyond the principal peaks (PP) region $\left(k>6 \AA^{-1}\right)$ are linked with second-neighbor correlations ${ }^{42}$. This is the case for a small contribution at $\simeq 6.5 \AA^{-1}$ that is barely visible in Fig. 3 except for GeTe but noticeable in Fig. $3 \mathrm{~b}$ for some other composition. It is reproduced from the simulation for amorphous GeTe only. In liquid state, it is possible to define an order parameter $S=S\left(k_{2}\right) / S\left(k_{1}\right)$ that allows distinguishing between an octahedral liquid $\left(S<1, \mathrm{Ge}_{15} \mathrm{Te}_{85}\right.$ for instance) and a tetrahedral one $\left(S>1, \mathrm{GeSe}_{2} \text { for instance }\right)^{44}$. Values of this parameter for the amorphous state are reported in Table I. Except for amorphous GeTe, for other compositions, $S$ is higher than 1 suggesting that these amorphous networks display an increased tetrahedral local order.

There are no main contribution arising from a given function $S_{n m}(k)$ as all weighted contributions $\langle f\rangle^{-2} c_{n} c_{m} f_{n} f_{m} S_{n m}(k)$ contribute in the range [0-0.3] to the total structure factor (Figure 4a). All correlations (chalcogen-chalcogen but also Ge-related) define the first PP at $k_{1}$ (green curves, Fig. 4a), whereas the secondary peak is dominated by contributions involving Ge atoms (Ge-Ge, $\mathrm{Ge}-\mathrm{Te}, \mathrm{Ge}-\mathrm{Se}$, broken green curve). A recent study associates the first PP and the second PP to some structural ordering ${ }^{43}$. The peak at $k_{2}$ is assumed to be a generic feature associated with nearest-neighbour contacts, and is therefore present in all amorphous materials. The peak amplitude at $k_{1}<k_{2}$ appears if directional character is present as in e.g. $\mathrm{GeSe}_{2}$, and this leads to the formation of tetrahedral motifs involving a second longer-length scale between such building blocks. Using this scheme, we understand that the importance of the secondary peak amplitude at $k_{2}$ results mainly from Ge contributions whose associated geometries are partly tetrahedral in GeTe and Te-containing systems and predominantly tetrahedral in GeSe. An inspection of this secondary peak with composition shows a moderate increase of the amplitude with Se content (Fig. 4b) and the increased contribution of Ge atoms to the secondary PP is essentially due to the reduction of Te atoms which have a larger form factor (taken here as $f_{i}=Z_{i}$ ).

\section{Real space properties}

We now turn to real space properties and represent in Figure 5 the calculated and measured pair correlation function $g(r)$. We note that the main features are reproduced from the simulation, from $\mathrm{GeTe}^{29}$ to those containing $\mathrm{Se}$ atoms, i.e. both positions and amplitudes of the two principal peaks at $r_{1} \simeq 2.40-2.59 \AA$ and $r_{2} \simeq 3.86-4.15 \AA$ (depending on experimental composition) are being recovered as we find numerically 2.43-2.63 $\AA$ and 3.86-4.15 $\AA$ for $r_{1}$ and $r_{2}$, respectively (Table I). We also remark that the evolution of the two peak positions found experimentally that is, a continuous decrease with Se content, is also reproduced from the DFT simulations. The origin of this trend is obviously driven by the reduction of the main bond lengths ( $\mathrm{Ge}-\mathrm{Se}$ is shorter than $\mathrm{Ge}-\mathrm{Te}$ ) and by the increasing density as one moves from GeTe to GeSe. Using total pair correlation functions $g(r)$ and the number densities $\rho_{0}{ }^{24}$ (see Table I ), it is possible to obtain a total coordination number $\mathrm{N}_{\text {tot }}(\mathrm{r})$ defined by:

$$
N_{t o t}=4 \pi \rho_{0} \int_{r_{0}}^{r_{\min }} r^{2} g(r) d r
$$

the lower integration bound $r_{0}$ being fixed to $2.2 \AA$ for all compositions (see Fig. 5). The values of the upper bound $r_{\text {min }}$ (given in Table I) are chosen as the value of the first minimum 

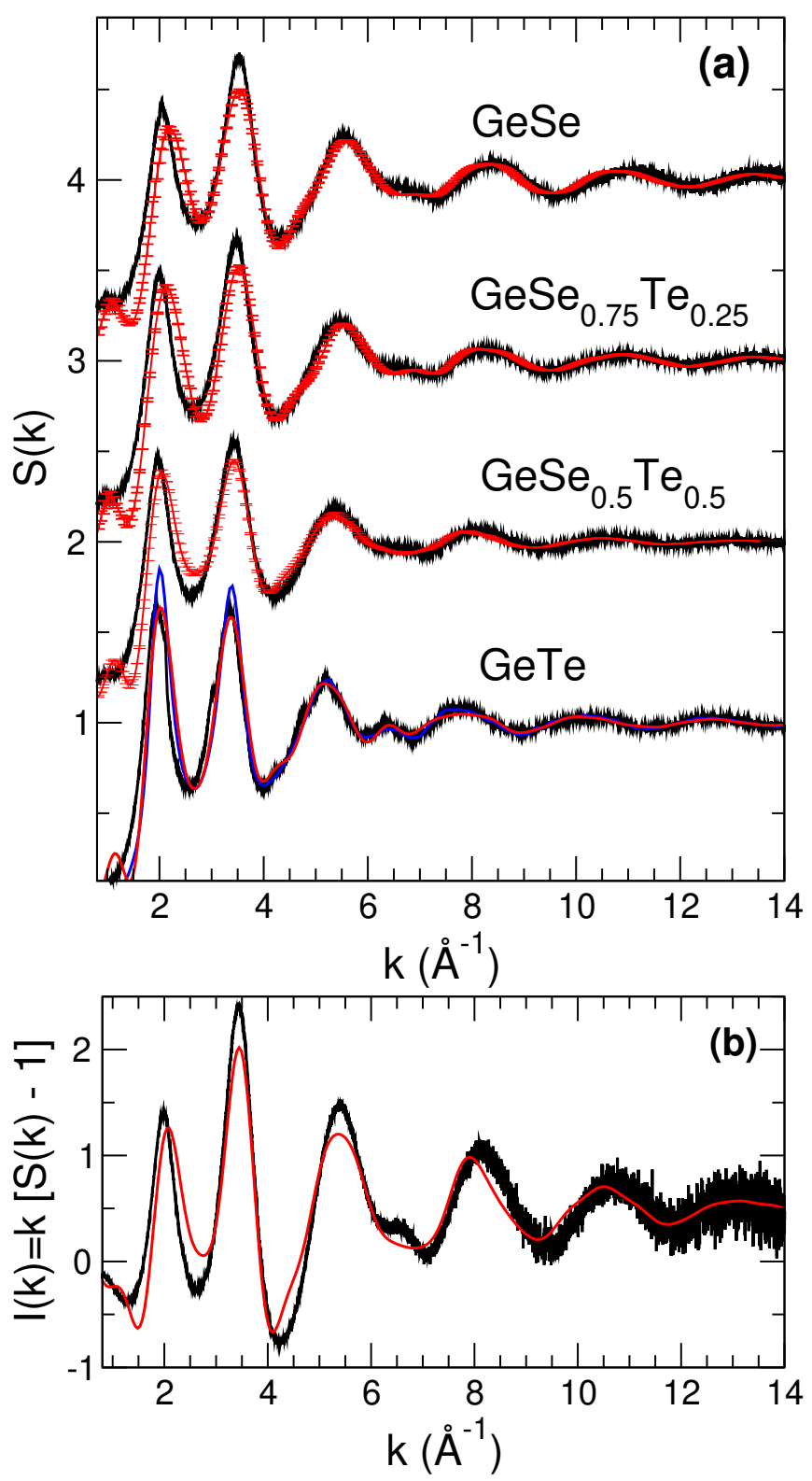

Figure 3: (a) Calculated structure factor $S(k)$ (red) compared to XRD experimental measurements (black). Results for GeTe are given $^{29}$. Error bars arise from the averages performed over the three independent quenches for the three present ternary compositions. The blue curve is from Piarristeguy et al. ${ }^{28}$. (b) Calculated interference function $I(k)$ (red) of $\mathrm{GeSe}_{0.50} \mathrm{Te}_{0.50}$ compared to XRD experimental measurements (black).

$\mathrm{r}_{\text {min }}$ of the function $\mathrm{r}^{2} \cdot g(r)$. Even if $\mathrm{N}_{t o t}$ has no real physical meaning since a binary/ternary alloy is studied here, it can be used as an indicator of the average structural changes undergoing as the Se content is changed. As seen in Table I, $\mathrm{N}_{t o t}$ increases with increasing the Se concentration into the base GeTe which might result from the substantial increase of the system density $\rho_{0}$ (Table I).
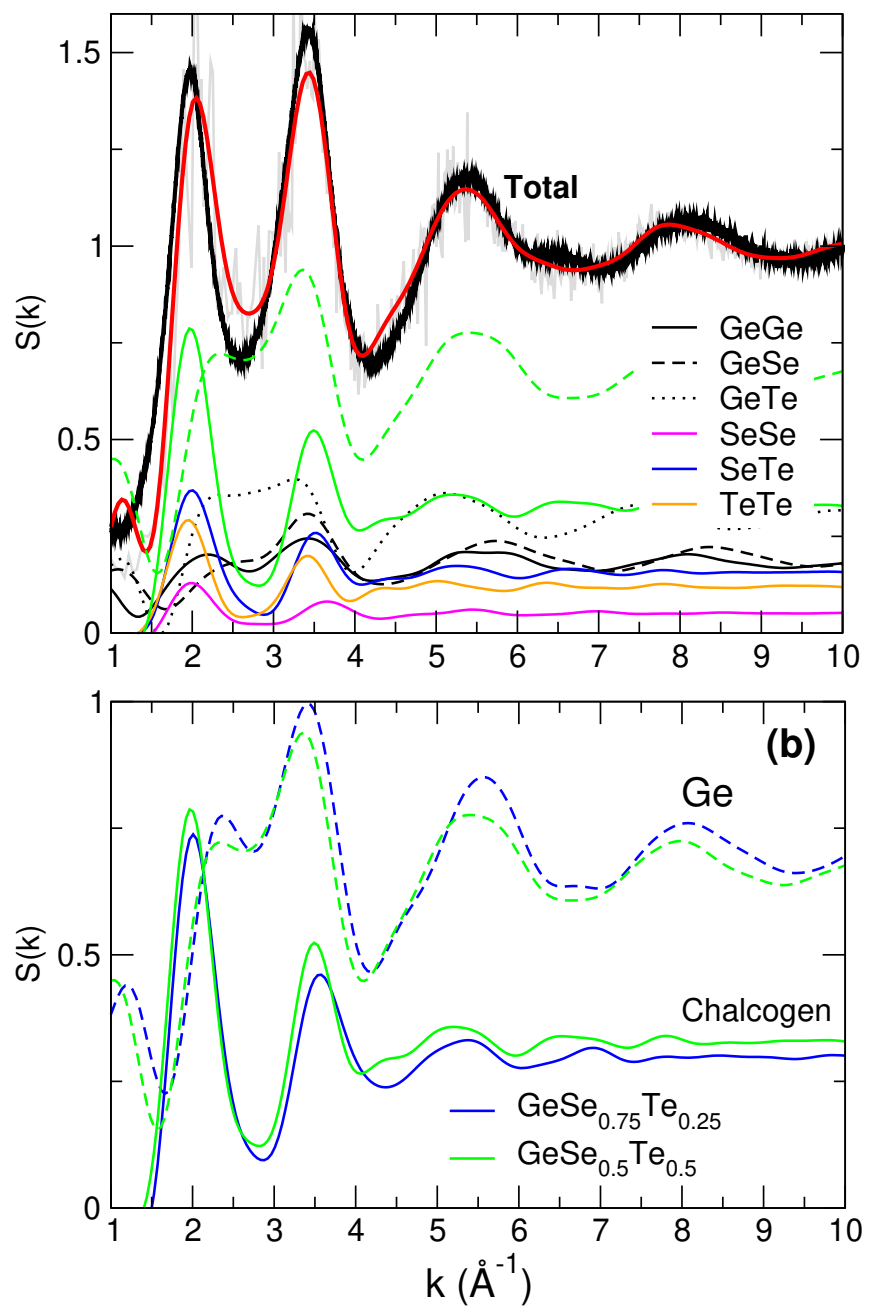

Figure 4: (a) Total calculated (red, equ. (3) and gray, equ. (4)) and measured (black) structure factor $S(k)$ of $\mathrm{GeSe}_{0.50} \mathrm{Te}_{0.50}$ (same as Fig. 3, red) and decomposition into weighted partials $S_{n m}(k)$. The broken and solid green curves correspond to total Ge and to (Te,Se) contributions, respectively. (b) Behavior of Ge and (Te,Se) contributions to $S(k)$ for $\mathrm{GeSe}_{0.50} \mathrm{Te}_{0.50}$ (same as Fig. 4a) and for $\mathrm{GeSe}_{0.75} \mathrm{Te}_{0.25}$ glasses.

\section{Partial pair correlations}

Figure 6 now represents the partial pair correlations for the four systems of interest. The study of amorphous GeTe has been extensively examined in ref. 29 and we just recall here the main features of the base network useful for the forthcoming discussion. We remind that although numerous simulation studies have been performed on amorphous $\mathrm{GeTe}^{45,46}$, recent investigations have emphasized the crucial role payed by dispersion forces in the DFT scheme in order to obtain accurate structure models which do not contain a spurious "bond-distance" problem (overestimation of Ge-Te bond distances). Using this improved MD scheme (DFT-D2), various scattering data can be accurately reproduced, i.e. Xray absorption spectroscopy (EXAFS ${ }^{47,48}$ ), anomalous X-ray scattering ${ }^{49}$ and X-ray scattering ${ }^{28}$ (see also Fig. 3a). The 


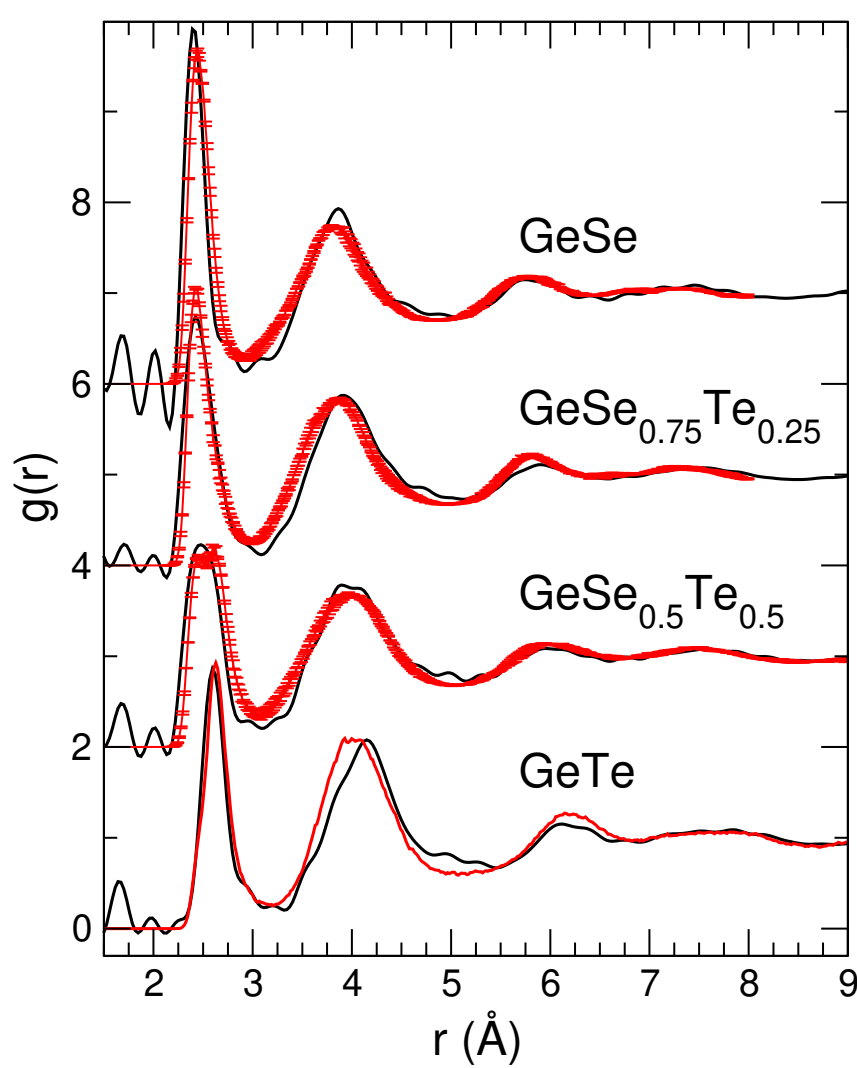

Figure 5: Calculated pair correlation function $g(r)$ (red) compared to XRD experimental measurements (black) of Ge-Se-Te glasses. Results for GeTe are given ${ }^{29}$. Error bars arise from the averages performed over the three independent quenches for the three present ternary compositions.

analysis of the partials reveals that GeTe contains predominantly homopolar Ge-Ge and heteropolar Ge-Te bonds characterized by distances of $2.50 \AA$ and $2.70 \AA$, respectively (experimentally $2.47 \AA$ and $2.62 \AA^{50}$ ). A small fraction of hompolar Te-Te bonds is found that lead to a prepeak in the $g_{T e T e}$ function at $2.89 \AA$ (Fig. 6c, inset). It should be noted that the fraction of such Ge-Ge bonds depends on the chosen electronic scheme ${ }^{51}$, an increased metallic character leading to a reduction of such defects in lighter chalcogenides. For the amorphous Tellurides, different simulation schemes have also emphasized the sensitivity of DFT electronic models on the Ge-Ge statistics ${ }^{52}$, but weak differences are found in Ge-Ge correlations between DFT-D2 and DFT-D3 schemes $^{53}$.

The substitution by Se atoms does not alter the short range structure associated with Ge atoms as noticed from the inspection of the corresponding partials (Fig. 6a,c,d), e.g. the function $g_{G e G e}$ displays the same principal peak at a distance of $2.50 \AA$ that is independent of Se content. An increased structuration of the base network manifests by a more pronounced minimum of $g_{G e G e}$ for $\mathrm{GeTe}$, however. The other pairs appear to be weakly sensitive to the Te/Se substitution.
Table II: Calculated partial coordination numbers $n_{n m}$ in the investigated systems. All have been calculated at the minimum $r_{\min }^{n m}$ of the corresponding partial pair correlation function $g_{n m}(r)$.

\begin{tabular}{|c|c|c|c|c|c|c|}
\hline System & $\mathrm{GeGe}$ & $\mathrm{GeSe}$ & $\mathrm{GeTe}$ & $\mathrm{SeSe}$ & SeTe & $\mathrm{TeTe}$ \\
\hline $\mathbf{G e T e}^{29}$ & 1.79 & & 2.20 & & & 0.28 \\
\hline EXAFS $^{50}$ & $1.57-1.89$ & & $1.38-1.47$ & & & \\
\hline $\mathbf{G e S e}_{0.50} \mathbf{T e}_{0.50}$ & 1.52 & 1.10 & 1.44 & - & - & 0.04 \\
\hline $\mathbf{G e S e}_{0.75} \mathbf{T e}_{0.25}$ & 1.47 & 1.77 & 0.65 & - & - & 0.05 \\
\hline GeSe & 1.34 & 2.46 & & - & & \\
\hline $1-\mathrm{GeSe}^{54}$ & 0.80 & 2.94 & & 0.01 & & \\
\hline $1-\mathrm{GeSe}^{55}$ & 0.80 & 3.2 & & 0.22 & & \\
\hline
\end{tabular}

\section{Coordinations}

Table II provides the detail of the coordination numbers using :

$$
n_{n m}=4 \pi \rho_{0} \int_{0}^{r_{\min }^{n m}} r^{2} g_{n m}(r) d r
$$

where $n_{n m}$ are partial coordination numbers, $g_{n m}(r)$ partial pair correlation functions and $\rho_{0}$ is given in Table I. It should first be reminded that for the base GeTe system, the reduced Te coordination number results from the incorporation of the DFT-D2 scheme ${ }^{29}$ that permits to reduce the Ge-Te bond length and the subsequent calculated $n_{G e T e}$ coordination. From Table II, we find $n_{G e}=n_{G e G e}+n_{G e T e}=3.99$ and $n_{T e}=n_{T e G e}+n_{T e T e}=2.48$ for Ge and Te atoms, respectively. Once Se atoms are added into the network, we first note the absence of Se-Se and Se-Te pairs as already acknowledged from the absence of a peak in the corresponding partial pair correlation functions at typical bonding distances (2.5-3.5 $\AA$, Fig. 6). It contrasts with the presence of remaining $\mathrm{Te}-\mathrm{Te}$ bonds present in amorphous GeTe (inset of Fig. 6c) but also in the ternary Ge-Se-Te glasses, and signals that the emerging GeSe network will be essentially dominated by $\mathrm{Ge}-\mathrm{Ge}$ and Ge-Se bonds.

With increasing Se content, the Ge-Ge binary coordination decreases from 1.79 (in the range of values determined from $\mathrm{EXAFS}^{50}$ ) to 1.34 for $\mathrm{GeSe}$, and for the Ge coordination $n_{G e}=n_{G e G e}+n_{G e T e}+n_{G e S e}$ we obtain 4.06 and 3.89 for $\mathrm{GeSe}_{0.50} \mathrm{Te}_{0.50}$ and $\mathrm{GeSe}_{0.75} \mathrm{Te}_{0.25}$, respectively (Table III). The corresponding Se coordination is found to be $n_{S e}=2.20$ and 2.36 for the same compositions.

The addition of Se into the base GeTe leads to a reduction of a higher coordinated defect geometries such as $\mathrm{Ge}^{V}$ which reduces from about $10 \%$ to only $2-3 \%$ for the ternary compositions Ge-Se-Te but increases finally to $8 \%$ for GeSe (Table III). This trend seems to parallel the one of $\mathrm{Ge}^{I I I}$ which 

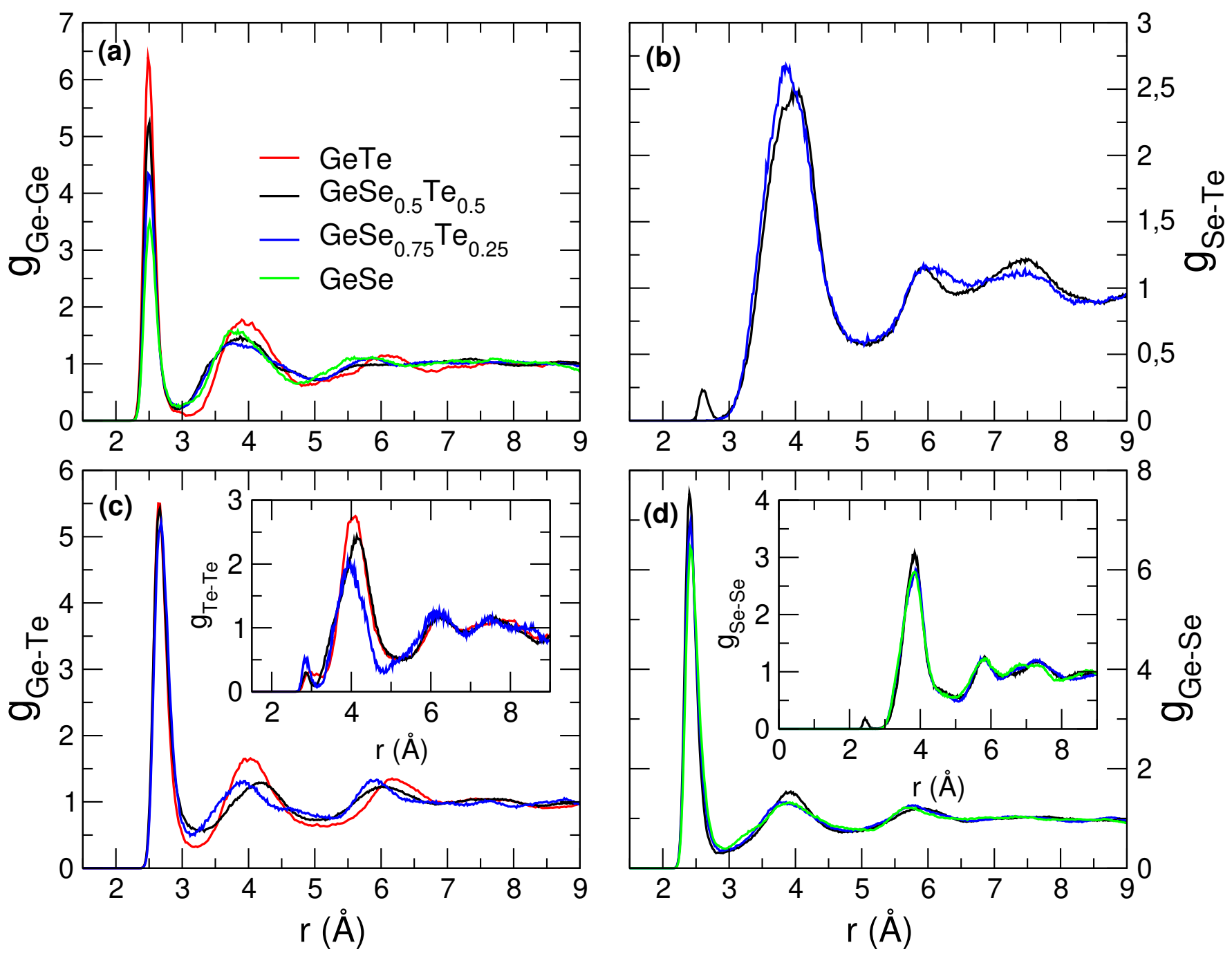

Figure 6: Calculated pair correlation function $g_{n m}(r)$ of Ge-Se-Te glasses. The insets in (c) and (d) show the $g_{T e T e}(r)$ and $g_{S e S e}(r)$ respectively.

Table III: Calculated coordination numbers $n_{m}$ in the investigated systems, together with coordination distribution and fraction $\eta_{T}$ of tetrahedral Ge. All have been calculated at the minima $r_{\min }^{m}$ of the corresponding partial pair correlation function $g_{n m}(r)$. A cutoff of $\sigma=12^{\circ}$ has been used for the calculation of $\eta_{T}$.

\begin{tabular}{|c|c|c|c|c|c|c|c|c|c|c|c|c|c|}
\hline System & $n_{G e}$ & $n_{S e}$ & $n_{T e}$ & $\eta_{T}(\%)$ & $\mathrm{Ge}^{I I I}(\%)$ & $\mathrm{Ge}^{I V}(\%)$ & $\mathrm{Ge}^{V}(\%)$ & $\operatorname{Te}^{I}(\%)$ & $\mathrm{Te}^{I I}(\%)$ & $\mathrm{Te}^{I I I}(\%)$ & $\operatorname{Se}^{I}(\%)$ & $\mathrm{Se}^{I I}(\%)$ & $\mathrm{Se}^{I I I}(\%)$ \\
\hline $\mathbf{G e T e}^{29}$ & 3.99 & & 2.48 & 64.7 & 8.3 & 81.2 & 10.1 & 0.7 & 57.1 & 39.7 & & & \\
\hline $\mathbf{G e S e}_{0.50} \mathbf{T e}_{0.50}$ & 4.06 & 2.20 & 2.92 & 66.1 & 17.0 & 79.9 & 3.1 & 4.0 & 56.2 & 39.8 & - & 84.0 & 16.0 \\
\hline $\mathbf{G e S e}_{0.75} \mathbf{T e}_{0.25}$ & 3.89 & 2.36 & 2.65 & 67.0 & 19.7 & 78.3 & 2.0 & - & 68.0 & 32.0 & - & 69.3 & 30.7 \\
\hline GeSe & 3.80 & 2.46 & & 74.0 & 11.8 & 80.2 & 8.0 & & & & - & 58.2 & 42.8 \\
\hline
\end{tabular}


behaves in the opposite direction (maximum of 3-fold $\mathrm{Ge}$ for the ternary compositions), so that the fraction of Ge defects remains essentially the same $(\simeq 20 \%)$ for all investigated compositions.

The coordination number of $\mathrm{Te}$ is dominated by 2 -fold species for all Te-based glasses and display a majority of 2fold coordination ranging from $57.1 \%$ up top $68.0 \%$ for GeTe and $\mathrm{GeSe}_{0.75} \mathrm{Te}_{0.25}$, respectively (Table III). A minority 3-fold coordination is also obtained (32-39.7\%) that is somewhat larger than the one determined for Se in GeSe (44.0\%).

The detail of the neighborhood of the Ge atoms appears also to be instructive. Here, we focus on the different $r$-fold Ge species $(r=3,4,5)$ and evaluate the number of $\mathrm{Ge}-\mathrm{Ge}, \mathrm{Ge}-\mathrm{Te}$ and Ge-Se bonds among the structure, given the information on the coordination statistics. Table IV represents such data for the four compositions of interest. Here the label $(g s)$ refers to the number $g$ of Ge neighbors around a $r$-folded $\mathrm{Ge}$, and to the number $s$ of Se neighbors, the number of Te atoms being given by $r-s-g$. For a 4-folded $\mathrm{Ge}\left(\mathrm{Ge}^{I V}\right)$, the structure (21) corresponds for instance to a $\mathrm{Ge}_{2} \mathrm{TeSe}-\mathrm{Ge}$ species.

The base GeTe network is dominated by Ge species having one (10), two (20) or three (30) homopolar Ge-Ge bonds with probability $36.2 \%, 42.8 \%$ and $21.5 \%$ for 4-fold Ge, respectively, whereas 5-fold Ge appears to attract a larger amount of Ge-Ge bonds (60.9 \% for (40) species), although his number has to be put in perspective with the small fraction of 5-fold Ge (10.1\%, Table III). Once Se is added into the network, we note a strong reduction for species having the largest number of $\mathrm{Ge}-\mathrm{Ge}$ bonds for $\mathrm{Ge}^{I V}$, i.e. (20) or (30) reduce substantially when changing from $\mathrm{GeTe}$ to $\mathrm{GeSe}_{0.50} \mathrm{Te}_{0.50}$, and the probability of finding a $\mathrm{Ge}_{3} \mathrm{Te}-\mathrm{Ge}$ species is very small $(0.7 \%)$ at this composition.

On the overall, for the compositions containing the three types of atoms, the dominant local structures appear to be made of mixed $\mathrm{Ge}$ species containing $\mathrm{Ge}$, Te and $\mathrm{Se}$ atoms without any preferential formation of "pure" species containing only one type of chalcogen atom, i.e. (21), (12) and (22) dominate for both $\mathrm{GeSe}_{0.50} \mathrm{Te}_{0.50}$ and $\mathrm{GeSe}_{0.25} \mathrm{Te}_{0.75}$, rather than e.g. (02), (03) or (20). This feature that can be also noticed from an inspection of the atomic snapshot (Fig. 1) that signals the presence of both $\mathrm{Te}$ and $\mathrm{Se}$ atoms in the vicinity of Germanium atoms. For the ternaries $\mathrm{GeSe}_{0.50} \mathrm{Te}_{0.50}$ and $\mathrm{GeSe}_{0.75} \mathrm{Te}_{0.25}$, we furthermore note the strong reduction of the (20) for in $\mathrm{Ge}^{I V}$ and $\mathrm{Ge}^{V}$ species, i.e. the substitution of Te by Se seems to essentially impact SRO that does not contain $\mathrm{Se}$ atoms at all. Instead, with growing Se content the dominant motif appears to be the (22) one that grows up to about $\simeq 45 \%$ for $\mathrm{Ge}^{I V}$, whereas it does not seem to be less frequent for 5-fold $\mathrm{Ge}\left(4.4 \%\right.$ in $\left.\mathrm{GeSe}_{0.75} \mathrm{Te}_{0.25}\right)$.

\section{Bond angle distributions}

Figure 7 represents the most significant bond angle distributions (BAD) of the different systems. Note that because the fraction of chalcogen triplet chains $X-X-X(X=\mathrm{Te}, \mathrm{Se})$ is less than $1 \%$, any combination of $(\mathrm{Te}, \mathrm{Se})$ lead to scattered and noisy distributions $P(\theta)$ which manifests e.g. in $\mathrm{Ge}-\mathrm{Se}-\mathrm{Te}$
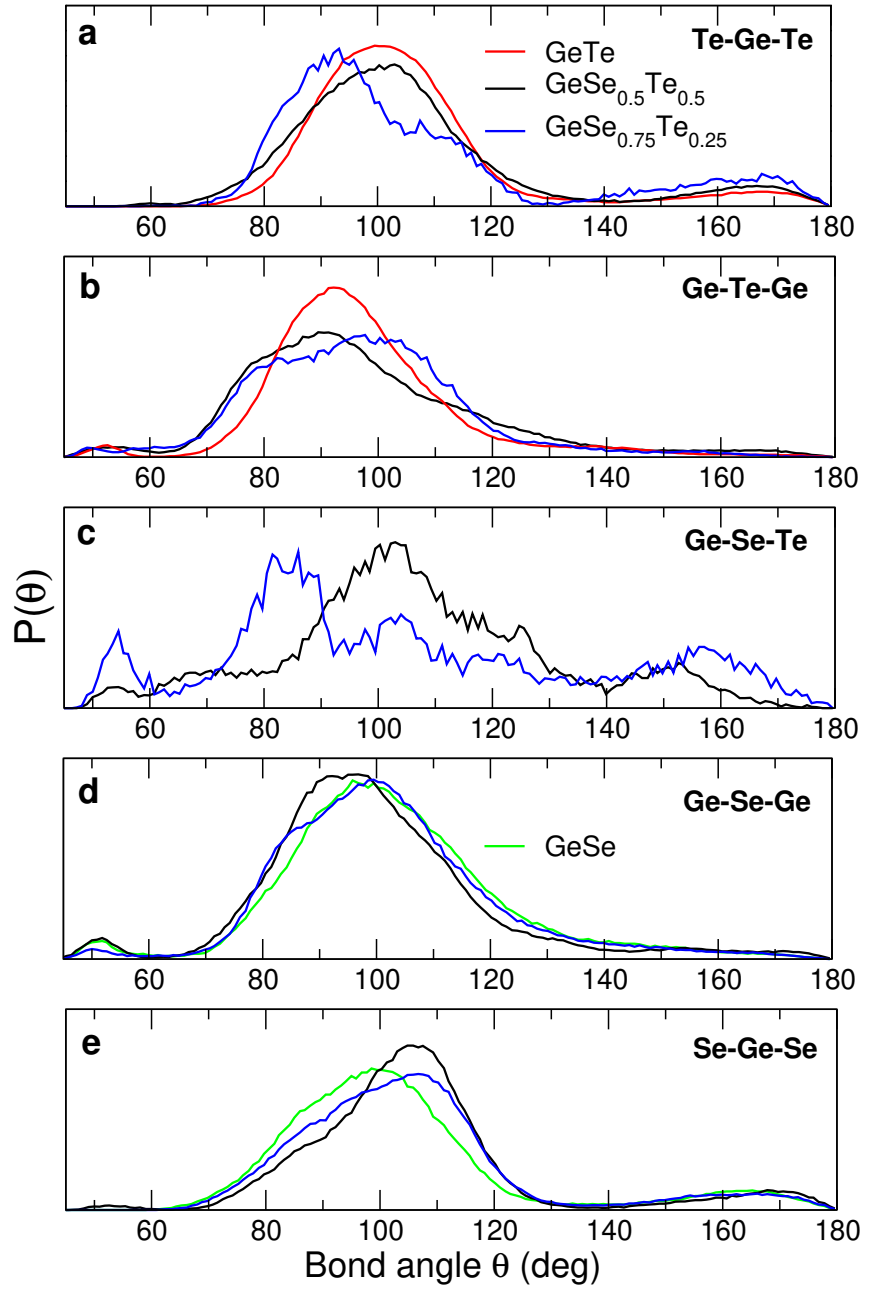

Figure 7: Select calculated bond angle distributions in Ge-Te-Se glasses: (a) Te-Ge-Te, (b) Ge-Te-Ge, (c) Ge-Se-Te, (d) Ge-Se-Ge and (e) Se-Ge-Se for different compositions : $\mathrm{GeTe}$ (red), $\mathrm{GeSe}_{0.50} \mathrm{Te}_{0.50}$ (black), $\mathrm{GeSe}_{0.75} \mathrm{Te}_{0.25}$ (blue) and $\mathrm{GeSe}$ (green).

(Fig. 7c) due to the very low fraction of chalcogen-chalcogen (i.e. Te-Se) bonds, as already detected from the bond analysis (Table III). This situation has been already noticed for the base GeTe amorphous system ${ }^{29}$. First, the local environment of Te atoms appears to be slightly changed with Se substitution although the BAD is always merely centred around the angle $90^{\circ}$ found in GeTe (Fig. 7b). A broadening of the distribution is found with increasing Se content, however. Conversely, the corresponding Ge-Se-Ge BAD (Fig. 7d) appears unchanged with composition. The evolution of Te-Ge-Te bond angles (Fig. 7a) appears more complex due to the mixed geometries (tetrahedral $(\mathrm{T})$ and octahedral $(\mathrm{O})$ ) that are already present in GeTe. This leads to a broad peak containing contributions at $90-100^{\circ}$ and $109^{\circ}$ in the Te-Ge-Te BAD (Fig. 7a) that is accompanied by a significant minority peak at $180^{\circ}$ which is indicative of a defect octahedral configuration for 4-fold $\mathrm{Ge}$. This behavior is at variance with the analogous Se-GeSe BAD (Fig. 7e) that clearly peaks at the tetrahedral angle of $109^{\circ}$. Using a constraint topological analysis, the population 
Table IV: Calculated fraction (\%) of species related to a $r$-fold coordinated Ge. The fractions are normalized with respect to a given $r$-fold population. The symbols $(g s)$ on the heading line refer to the number of $\mathrm{Ge}$ and Se atoms around a $r$-fold coordinated Ge, respectively, e.g. 31 refers to $3 \mathrm{Ge}, 1 \mathrm{Se}$ and $r$ - $g$-s=r-4 Te around a $r$-fold Ge atom.

\begin{tabular}{|c|c|c|c|c|c|c|c|c|c|c|c|c|c|c|c|c|c|c|}
\hline & System & 10 & 01 & 11 & 20 & 02 & 21 & 12 & 22 & 30 & 03 & 13 & 31 & 32 & 23 & 40 & 14 & 41 \\
\hline $\mathrm{Ge}^{I I I}$ & $\begin{array}{l}\mathrm{GeTe} \\
\mathrm{GeSe}_{0.50} \mathrm{Te}_{0.50} \\
\mathrm{GeSe}_{0.75} \mathrm{Te}_{0.25} \\
\mathrm{GeSe}\end{array}$ & $\begin{array}{c}69.3 \\
24.7 \\
-\end{array}$ & $\begin{array}{l}3.7 \\
6.4\end{array}$ & $\begin{array}{l}13.5 \\
27.5\end{array}$ & $\begin{array}{c}28.2 \\
6.5 \\
21.1\end{array}$ & $\begin{array}{l}6.5 \\
8.8\end{array}$ & $\begin{array}{l}11.2 \\
11.1 \\
26.4\end{array}$ & $\begin{array}{l}32.1 \\
19.9 \\
44.0\end{array}$ & & $\begin{array}{l}2.5 \\
1.9 \\
5.3 \\
-\end{array}$ & 29.6 & & & & & & & \\
\hline $\mathrm{Ge}^{I V}$ & $\begin{array}{l}\mathrm{GeTe} \\
\mathrm{GeSe}_{0.50} \mathrm{Te}_{0.50} \\
\mathrm{GeSe}_{0.75} \mathrm{Te}_{0.25} \\
\mathrm{GeSe}\end{array}$ & $\begin{array}{c}36.2 \\
3.7 \\
-\end{array}$ & - & $\begin{array}{c}9.1 \\
14.8\end{array}$ & $\begin{array}{c}42.8 \\
16.5 \\
5.4\end{array}$ & $\begin{array}{c}2.7 \\
-\end{array}$ & $\begin{array}{l}17.2 \\
11.7\end{array}$ & $\begin{array}{c}20.9 \\
7.4\end{array}$ & $\begin{array}{l}23.3 \\
44.7 \\
45.2\end{array}$ & $\begin{array}{c}21.5 \\
0.4 \\
0.7\end{array}$ & $\begin{array}{l}0.2 \\
2.7\end{array}$ & $\begin{array}{c}5.4 \\
9.7 \\
51.8\end{array}$ & $\begin{array}{l}0.2 \\
2.7 \\
3.0\end{array}$ & & & $\begin{array}{c}1.1 \\
0.2 \\
- \\
-\end{array}$ & & \\
\hline $\mathrm{Ge}^{V}$ & $\begin{array}{l}\mathrm{GeTe} \\
\mathrm{GeSe}_{0.50} \mathrm{Te}_{0.50} \\
\mathrm{GeSe}_{0.75} \mathrm{Te}_{0.25} \\
\mathrm{GeSe}\end{array}$ & $\begin{array}{c}1.1 \\
4.1 \\
-\end{array}$ & - & $\begin{array}{l}2.1 \\
0.2\end{array}$ & $\begin{array}{c}27.6 \\
21.8 \\
-\end{array}$ & - & $\begin{array}{c}4.6 \\
10.1\end{array}$ & $\begin{array}{c}1.6 \\
12.1\end{array}$ & $\begin{array}{c}13.1 \\
4.4\end{array}$ & $\begin{array}{c}10.4 \\
10.4 \\
5.4\end{array}$ & - & $\begin{array}{c}15.3 \\
6.2\end{array}$ & $\begin{array}{l}2.7 \\
4.7\end{array}$ & $\begin{array}{c}5.7 \\
17.0 \\
16.3\end{array}$ & $\begin{array}{l}15.2 \\
15.1 \\
54.9\end{array}$ & $\begin{array}{c}60.9 \\
0.2 \\
-\end{array}$ & $\begin{array}{c}3.0 \\
2.7 \\
26.6\end{array}$ & $\begin{array}{c}- \\
19.8 \\
2.2\end{array}$ \\
\hline
\end{tabular}

of tetrahedra or the contribution of the two species can be separated and for amorphous GeTe it was found that the fraction $\eta_{T}$ of Ge tetrahedra was about $65 \%$ (Table III). This value is much larger than previously reported and highly sensitive to i) the electronic scheme used for the DFT that impacts the Ge-Te bond length ${ }^{56}$ and the subsequent calculated $\eta_{T}$, and ii) the method used to determine such population (for a discussion, see Ref. 57). It is also important to stress that the most rigorous method based on topological constraints is compatible with an evaluation of $\eta_{T}$ from Mössbauer experiments in $\mathrm{Ge}-\mathrm{Te}, \mathrm{Si}_{-} \mathrm{Te}^{57}$ and Ge-Sb-Te ${ }^{58}$.

An inspection of the additional panels provided in Figure 8 finally signals that the presence of homopolar Ge-Ge bonds does not affect the angular properties of the structure, and this becomes particularly obvious as one compares for e.g. the intermediate composition $\mathrm{GeSe}_{0.50} \mathrm{Te}_{0.50}$, the distributions Se$\mathrm{Ge}-\mathrm{Te}, \mathrm{Ge}-\mathrm{Ge}-\mathrm{Se}$ and $\mathrm{Ge}-\mathrm{Ge}-\mathrm{Te}$. Concerning the latter, it has been shown ${ }^{59}$ that the presence of homopolar Ge-Ge was promoting the population of Ge tetrahedra in phase change Tellurides, and the present results of Figure $8 \mathrm{~b}$ indicates, indeed a more sharper peak around $109^{\circ}$ that progressively broadens as the Se content is increased, consistently with the growth of the calculated fraction $\eta_{T}$ (Table III).

\section{ELECTRONIC PROPERTIES}

From four select configurations per composition of the amorphous phases, we have performed the calculation of their electronic structures which are displayed in Figs. 9 and 10. We first note that for GeTe, the calculated band structure reproduces rather well experimental results obtained from Xray photoemission spectroscopy (XPS ${ }^{60,61}$ ), and the band gap

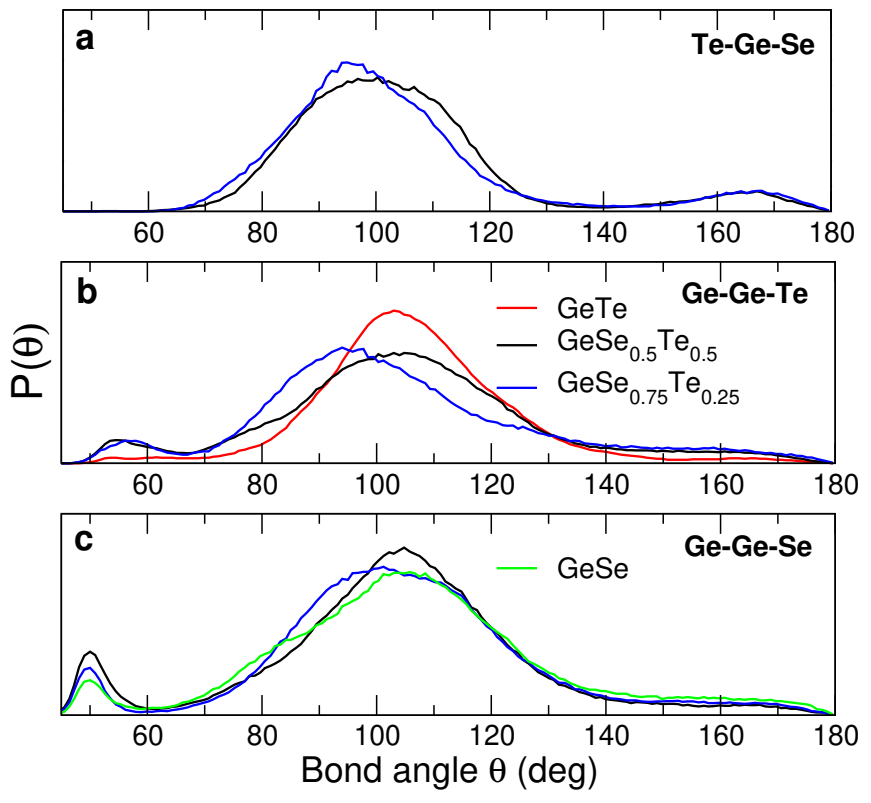

Figure 8: Select calculated Ge-centred bond angle distributions : (a) Te-Ge-Se, (b) Ge-Ge-Te and (c) Ge-Ge-Se for different compositions: $\mathrm{GeTe}$ (red), $\mathrm{GeSe}_{0.50} \mathrm{Te}_{0.50}$ (black), $\mathrm{GeSe}_{0.75} \mathrm{Te}_{0.25}$ (blue) and GeSe (green).

is found to be of about $0.7 \mathrm{eV}$, consistently with previous DFT calculations ${ }^{62}$ which are close to the experimental estimates (0.75-0.85, see Refs. 63-65). Early XPS studies ${ }^{60,66,67}$ have emphasized the important difference in density of states of amorphous and crystalline GeTe that lead to a semi- or p-type extrinsic semiconducting behavior, respectively. The 


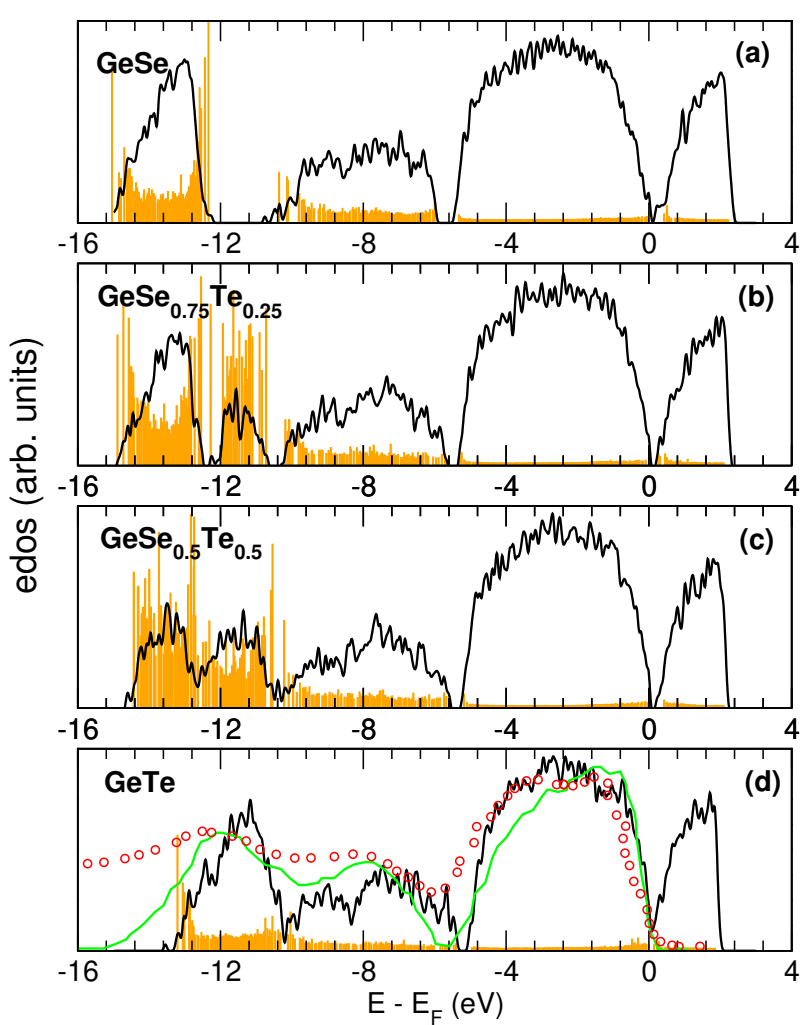

Figure 9: Calculated electronic density of states (EDOS) and inverse participation ratio (IPR, orange) of Ge-Te-Se glasses. Note that same scale is used for the IPR axis in all panels. In panel (d), experimental XPS results for amorphous GeTe are provided (red circles ${ }^{60}$ and green curve ${ }^{61}$ ).

calculated Fermi energy $E_{F}$ increases linearly with Se content from $0.56(7) \mathrm{eV}$ for GeTe (experimentally $0.31 \mathrm{eV}^{68}$ ) up to 2.38(6) eV for GeSe. The double peak observed in the p-band structure with a minimum at around $-2.5 \mathrm{eV}$ (red circles ${ }^{60}$, Fig. 9d) was assigned to presence of long-range order but has not been detected in more recent studies ${ }^{61}$. This double peak is not obtained from the present simulations. The overall form of the valence band of GeTe represented in Fig. 9d can be understood in terms of a contribution of s-orbitals of $\mathrm{Ge}$ and $\mathrm{Te}$ centred at -8.0 and $-11.5 \mathrm{eV}$, respectively (Figure 9), together with a broad band close to the Fermi level that is dominated by p-orbitals of Te atoms.

As noticed from Fig. 9a, the profile of amorphous GeSe turns out to be radically different because of the presence of a large gap between -12.0 and $-9.5 \mathrm{eV}$ that separates s-band arising from Se from the s-band of Ge.

The projected DOS furthermore shows that for the two ternary Ge-Te-Se compositions, the alteration mostly occurs in the low energy bands corresponding to s-contributions from $\mathrm{Te}$ and Se atoms, centred at $-11.6 \mathrm{eV}$ and $-13.5 \mathrm{eV}$, respectively. While the $\mathrm{GeSe}_{0.50} \mathrm{Te}_{0.50}$ compound exhibits a near continuous band distribution covering s-orbitals of all involved atoms from $-5.7 \mathrm{eV}$ down to $-15 \mathrm{eV}$, the Se-rich composition $\left(\mathrm{GeSe}_{0.75} \mathrm{Te}_{0.25}\right)$ exibits well separated bands (s-Se, $\mathrm{s}-\mathrm{Te}, \mathrm{s}-\mathrm{Ge}$ ) with gaps that are of about $0.5 \mathrm{eV}$ for each, and

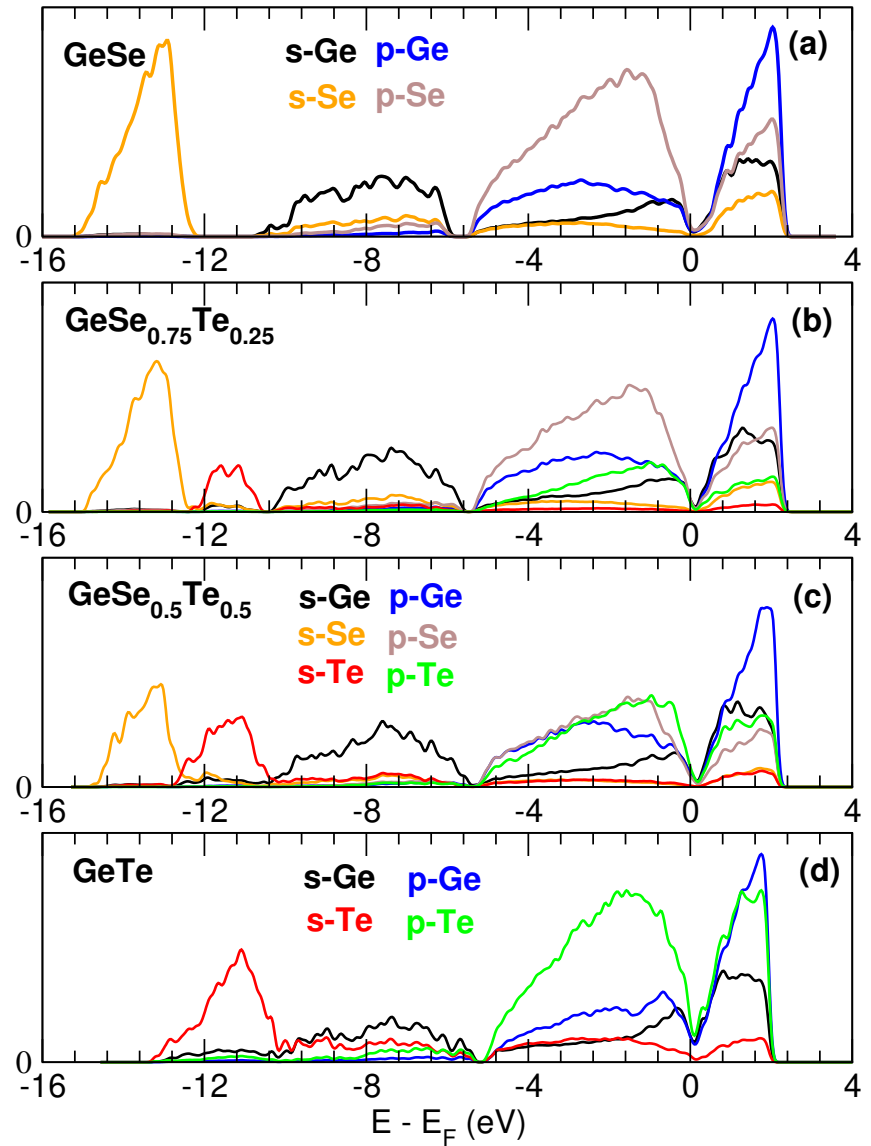

Figure 10: Calculated electronic density of states (EDOS) of GeTe-Se glasses. Partial contributions (atoms, orbitals) are given by colored curves.

this signals the emerging electronic structure of amorphous GeSe. As noticed from Fig. 10, the conduction band is dominated by contributions from p-orbitals, both $\mathrm{Ge}$ and Te.

An addition analysis using the inverse participation ratios (IPR, orange in Fig. 9) defined by :

$$
I P R=\frac{\int d \mathbf{r}|\Psi(\mathbf{r})|^{4}}{\left(\int d \mathbf{r}|\Psi(\mathbf{r})|^{2}\right)^{2}}
$$

permits to measure the degree of localized orbitals as large value are usually indicative of localization around specific bonds, i.e. IPR $\rightarrow 0$ for a fully delocalized state (conductor) and IPR $\rightarrow 1$ for a pure state. An combined inspection of GeTe and GeSe suggests a much more localized bonding for the latter which is consistent with its increased covalent character. It manifests by large IPR values (0.2) close to the edges of the $\mathrm{s}$-band and to a lesser extent for energies at about $-11 \mathrm{eV}$ in the s-Ge band.

The addition of Se atoms into the base GeTe system now reveals that the localization of the bonding will increase as suggested by the large contributions over the entire s-Te and s-Se bands (Figs. 9(b)-(c)), also indicating an increased localized nature of the $\mathrm{KS}$ states in the region ranging from $-15 \mathrm{eV}$ up to the lower edge of the s-band of Ge $(\simeq-9 \mathrm{eV})$. The ternary compositions appear, thus, to have a near covalent character that 
is clearly increased with respect to the starting GeTe composition. Clearly the Te-related bands are much more localized once Se atoms are added into the network.

On the overall, the trend observed with Se addition apperas to be compatible with a loss in metallicity that can be quantified by a parameter introduced recently ${ }^{69}$ that defines a material metallicity from the inverse of an average electronegativity :

$$
M_{P}^{-1}=\sum_{k} x_{k} \chi_{k}
$$

where $x_{k}$ is the atomic fraction of each component $k$ and $\chi_{k}$ is the corresponding Pauling electronegativity ${ }^{70}$. An evaluation of such a metallicity parameter $M_{P}$ indicates, indeed, that it decreases continuously from $M_{P}=0.48(7)$ for GeTe to $\mathbf{0 . 4 3 ( 9 )}$ for $\mathrm{GeSe}$, the ternary compositions leading to $M_{P}=0.46(1)$ and $0.45(0)$ for $\mathrm{GeSe}_{0.50} \mathrm{Te}_{0.50}$ and $\mathrm{GeSe}_{0.75} \mathrm{Te}_{0.25}$, respectively. The values of $0.48-0.49$ are those where most of the PCM are found, i.e. those lying on the pseudo-join GeTe-Sb${ }_{2} \mathrm{Te}_{3}$ and (Ag,In) doped $\mathrm{Sb}_{2} \mathrm{Te}_{3}{ }^{69}$, whereas Se-based Tellurides (e.g. the series $\mathrm{Se}_{x} \mathrm{Te}_{100-x}{ }^{71}$ ) display a lower $M_{P}$ due to the presence of the more electronegative Selenium.

The loss in metallicity also might improve the glassforming tendency of such materials as Se-containing liquids are usually known to form glasses over extended ranges in composition ${ }^{5}$. This comment appears to be in line with the conclusions of a recent study on the role of chemical bonding (from metallic to covalent) on the vitrification properties in Ge-Te-Se ${ }^{72}$. Here, it was found that at a fixed but standard heating rate, only Ge-Se compositions on the GeSe-GeTe pseudo-binary line could show a clear glass transition, enabling the determination of a characteristic temperature.

\section{VIBRATIONAL PROPERTIES}

We now turn to vibrational properties. Figure 11a represents the vibrational density of states (VDOS) defined by the Fourier transform of the velocity-velocity autocorrelation function $\langle\mathbf{v}(t) \cdot \mathbf{v}(0)\rangle$ :

$$
g(\omega)=\frac{1}{3 N k_{B} T} \sum_{j=1}^{N} \int_{0}^{\infty}\left\langle\mathbf{v}_{j}(t) \cdot \mathbf{v}_{j}(0)\right\rangle e^{i \omega t} d t
$$

where $N, k_{B}$ and $T$ are the total number of atoms, the Boltzmann constant and the temperature respectively. The VDOS of the base system (GeTe) consists in two broad contributions centred at $70 \mathrm{~cm}^{-1}$ and $220 \mathrm{~cm}^{-1}$ (Fig. 11e) and can be associated with vibrations related with $\mathrm{Te}$ and $\mathrm{Ge}$, respectively. The lower frequencies are usually associated with the chalcogen atoms ${ }^{73}$ and a previous DFT study ${ }^{74,75}$ of amorphous GeTe has indicated that the VDOS has also a two-component structure and expands up to $250 \mathrm{~cm}^{-1}$. As one evolves from amorphous GeTe to GeSe, an slight shift to larger frequencies is acknowledged for the high frequency band and for GeSe,

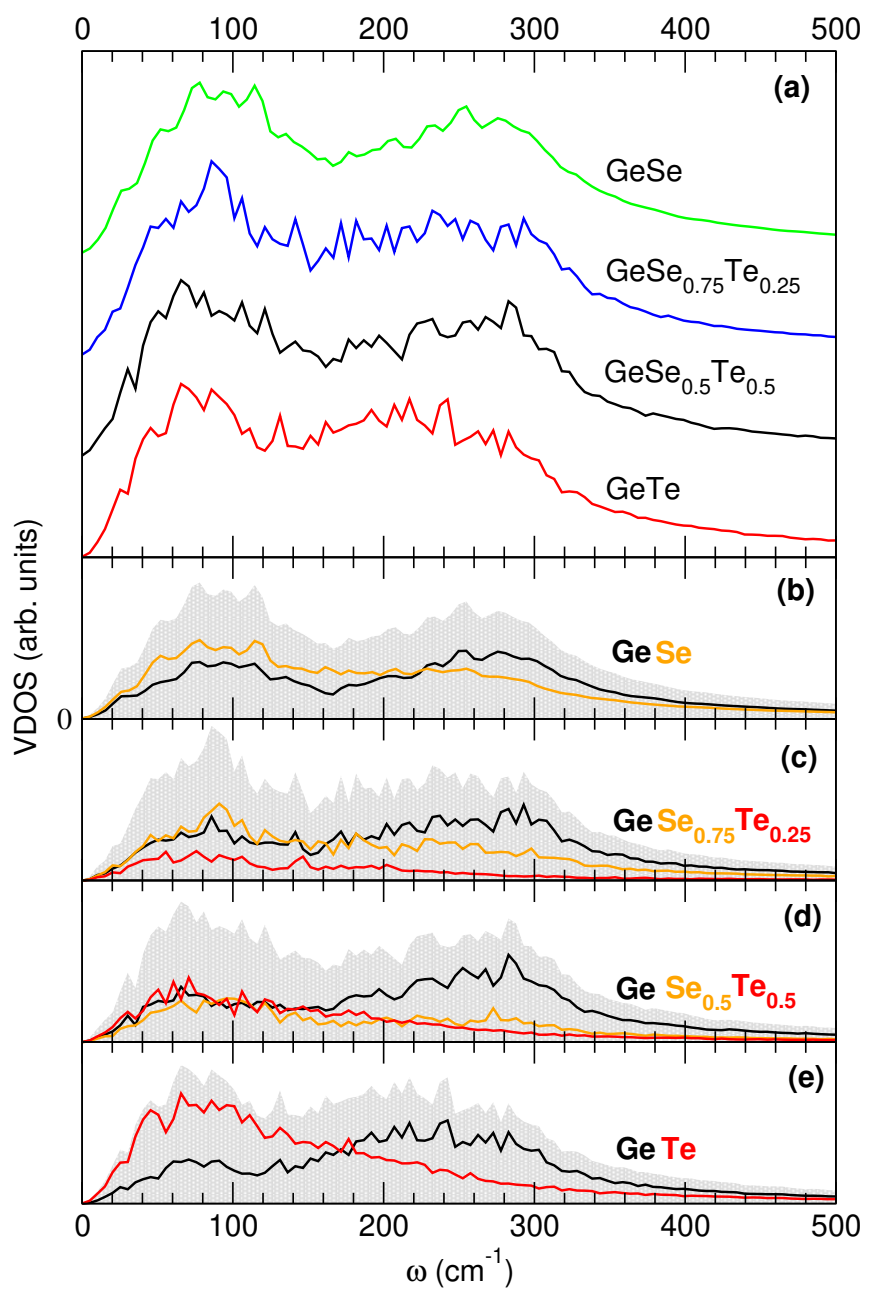

Figure 11: Calculated vibrational density of states (VDOS) of amorphous Ge-Te-Se compounds. (a) Total VDOS. (b)-(e) Total VDOS for each composition (shaded gray) together with partial contributions from Ge (black), Se (orange) and Te (red).

this band is centred at about $270 \mathrm{~cm}^{-1}$, and more well separated from the low frequency band that has also shifted to larger $\omega$ a lower extent.

The substitution of Te by Se atoms leads to a more balanced contribution at low frequency as Se and Ge now both contribute to the VDOS of amorphous GeSe for the low frequency band (Fig. 11b), the ternary compositions having a nearly equal contribution for $\omega<150 \mathrm{~cm}^{-1}$, whereas the high frequency band continues to be dominated by Ge-related vibrations.

In amorphous GeTe, the broad band at large frequency seem to be correlated with two typical peaks in Raman scattering ${ }^{64}$ at 122 and $162 \mathrm{~cm}^{-1}$, whereas the band mostly linked with Te (Fig. 11e) at $\omega<120 \mathrm{~cm}^{-1}$ are usually associated with Te and particularly 3 -fold Te $\left(\omega=83 \mathrm{~cm}^{-1}\right)$ that has been inferred from $a b$ initio simulations and empirical bond polarizability models $^{76}$. A recent study of amorphous GeTe-GeSe systems ${ }^{77}$ focuses only on the Raman properties induced by the substitution of Se into crystalline GeTe and highlights essentially the 
Table V: Select mode frequencies $\omega_{k}$ (in $\mathrm{cm}^{-1}$ ) of Ge-Te-Se DFT optimized clusters. The values in bracketts indicate angular excursions Te-Ge-Te for the select case of $\mathrm{GeSe}_{1} \mathrm{Te}_{3} \mathrm{H}_{4}$.

\begin{tabular}{|c|c|c|c|c|c|c|c|}
\hline System & $\omega_{1}$ & $\omega_{2}$ & $\omega_{3}$ & $\omega_{4}$ & $\omega_{5}$ & $\omega_{6}$ & $\omega_{7}$ \\
\hline T-GeTe ${ }_{4} \mathbf{H}_{4}$ & $\begin{array}{l}74.0 \\
\text { BB }\end{array}$ & $\begin{array}{c}75.0 \\
\text { symetric }\end{array}$ & $\begin{array}{c}86.8 \\
\text { BB } \\
\text { breathing }\end{array}$ & $\begin{array}{c}192.6 \\
\text { asymetric }\end{array}$ & $\begin{array}{c}231.1 \\
\text { asymetric } \\
\text { BS }\end{array}$ & $\begin{array}{c}248.6 \\
\text { asymetric } \\
\text { BB }\end{array}$ & $\begin{array}{c}307.7 \\
\text { BS }\end{array}$ \\
\hline T-GeSe $\mathbf{T e}_{3} \mathbf{H}_{4}$ & $\begin{array}{c}76.7 \\
\left(88-131^{\circ}\right)\end{array}$ & $\begin{array}{c}84.2 \\
\left(100-118^{\circ}\right)\end{array}$ & $\begin{array}{c}94.9 \\
\left(90-121^{\circ}\right)\end{array}$ & $\begin{array}{c}197.2 \\
\left(73-133^{\circ}\right)\end{array}$ & $\begin{array}{c}244.8 \\
\left(93-120^{\circ}\right)\end{array}$ & $\begin{array}{c}267.3 \\
\left(102-114^{\circ}\right)\end{array}$ & $\begin{array}{c}308.6 \\
\left(97-127^{\circ}\right)\end{array}$ \\
\hline $\mathbf{T}-\mathbf{G e S e}_{2} \mathbf{T e}_{2} \mathbf{H}_{4}$ & 82.9 & 87.1 & 103.2 & 213.4 & 262.4 & 268.9 & 308.8 \\
\hline $\mathbf{T}-\mathbf{G e S e}_{4} \mathbf{H}_{4}$ & 83.8 & 88.1 & 105.3 & 215.4 & 290.6 & 298.1 & 357.2 \\
\hline O-GeTe ${ }_{4} \mathbf{H}_{4}$ & 74.0 & 75.1 & 86.8 & 192.6 & 231.1 & 248.6 & 307.6 \\
\hline
\end{tabular}

transformation of rhombohedral GeTe into the orthorhombic GeSe phase.

To further decode vibrational properties, we have performed DFT calculations on Ge-Se-Te clusters representative of the short-range order of the network structure. The harmonic frequencies were calculated on optimized structures using as starting configurations perfect tetrahedra $\left(\mathrm{T}-\mathrm{GeX}_{4} \mathrm{H}_{4}\right)$ and defect octahedra $\left(\mathrm{O}-\mathrm{GeTe}_{4} \mathrm{H}_{4}\right)$, the number of Se atoms being changed on the former (from 0 up to 4). Corresponding eigenfrequencies are provided in (Table V). These concentrate on the frequency range $\left(70-300 \mathrm{~cm}^{-1}\right)$ where Raman and IR active modes are present ${ }^{60}$. In amorphous GeTe, typical observed modes are associated with asymetric bondstretching motion $\left(218 \mathrm{~cm}^{-1}\right)$ of tetrahedral $\mathrm{GeTe}_{4}, \mathrm{Te}$ vibrations $\left(158 \mathrm{~cm}^{-1}\right)$ and a shoulder peak at $165 \mathrm{~cm}^{-1}$. A more recent DFT-based analysis ${ }^{76}$ has provided some support to these claims as the spectrum above $190 \mathrm{~cm}^{-1}$ appears to be dominated by tetrahedral structures, while the most prominent peaks around 120 and $165 \mathrm{~cm}^{-1}$ arise mainly from vibrations of atoms in defective octahedral sites.

This analysis reveals that a certain number of frequencies are not sensitive to composition and this appears to be valid for the lowest frequency $\left(\omega_{1} \simeq 74-83 \mathrm{~cm}^{-1}\right)$, and to lesser extent to certain intermediate frequencies $\left(\omega_{2}, \omega_{3}, \omega_{4}\right)$ that are associated with bending and breathing motions. The replacement of Te by Se induces a slight stiffening of the motion as manifested by e.g. asymetric bond-streching vibrations which increase continuously from $\omega_{5}=231.1 \mathrm{~cm}^{-1}$ for $\mathrm{T}-\mathrm{GeTe}_{4} \mathrm{H}_{4}$ up to $290.6 \mathrm{~cm}^{-1}$ for $\mathrm{T}-\mathrm{GeSe}_{4} \mathrm{H}_{4}$, this tendency being also observed for $\omega_{6}$ and $\omega_{7}$, and responsable with the shift to larger $\omega$ of the high frequency band of the VDOS with Se substitution (Fig. 11a). Using a nearest-neighbor central-force model, Sen and Thorpe ${ }^{78}$ have proposed a relation $\omega=\sqrt{\beta / m} \cos \theta / 2$, where $\beta$ is a restoring force constant, $\theta$ the involved angle and $m$ an effective mass for the vibrating structures such as the oxygen mass in an $\mathrm{Si}-\mathrm{O}-\mathrm{Si}$ motion in silica. The analogy leads here to a Ge-X-Ge motion
( $\mathrm{X}=\mathrm{Te}, \mathrm{Se}$ ) that indicates that the replacement of Te by the less heavier Se atom clearly must induce a shift of the VDOS and Raman/IR bands to higher frequency when changing amorphous GeTe to GeSe.

\section{CONCLUSION}

Chalcogenides using $\mathrm{Ge}, \mathrm{Se}$, and Te represent materials of special importance given their possibilities in optoelectronic applications. Such applications are driven by fundamental properties arising from structure and chemical bonding.

Here, we have focused on the structural, electronic and vibrational properties of four compositions in the amorphous Ge-Se-Te ternary undergoing a $\mathrm{Te} \leftrightarrow$ Se substitution by combining molecular dynamics simulations and X-ray diffraction experiments. The base system is GeTe that has been previously characterized and the starting simulated structure appears to be very realistic as thoroughly demonstrated in Ref. 29. The study of all compositions indicates that both the experimental pair correlation function in real space, and the structure factor in reciprocal space can be reproduced from molecular simulations with a very satisfying accuracy. This permits to decode structural features that cannot be accessed from experiments.

The molecular simulations reveal, indeed, that the networks are dominated by four-fold Ge, 2-fold Se and a mixture of 2and 3-coordinated Te as in archetypal binary tellurides ${ }^{29,57}$. Homopolar bonds are only found for Ge-Ge and a small fraction of Te-Te pairs and signatures of other chalcogenchalcogen homopolar bonds (Te-Se, Se-Se) are barely present. The rest of the network is dominated by Ge-Se, and Ge-Te bonds that are found in diffreent populations : tetrahedral $(\mathrm{T})$ and octahedral $(\mathrm{O}) \mathrm{Ge}$ to a dominant tetrahedral network in $\mathrm{GeSe}(74 \%)$ that contains large bond-bending motions driven by the stress-release of highly cross-linked amorphous networks. While the local structure of the GeTe compound ap- 
pears to share essentially one or two Ge atoms around the $r$ folded $\mathrm{Ge}$ species, the addition of Se leads to mixed geometries that have been characterized and can connect e.g. two Se and two Te atoms to a 4-fold Germanium atom.

Regarding chemical bonding, we first reproduce correctly the electronic density of states accessed from XPS measurements with corresponding $4 \mathrm{~s}$ and $4 \mathrm{p}$ bands that evolve from $\mathrm{GeTe}$ and then demonstrate that the addition of Selenium increases the covalent character of Te-based bonds as the localization of corresponding 4s-orbitals is substantially increased for the considered ternary compositions $\mathrm{GeSe}_{0.5} \mathrm{Te}_{0.5}$ and $\mathrm{GeSe}_{0.75} \mathrm{Te}_{0.25}$. This indicates that such materials evolve from a bonding that is in between the metallic and the covalent bond in GeTe towards nearly full covalent character at already
$50 \%$ substituted Se. These properties might be employed to design dedicated opto-electronic materials.

\section{Acknowledgements}

H. M. Flores-Ruiz gratefully acknowledges the computing time granted by LANCAD and CONACYT on the supercomputer Yoltla/Miztli/Xiuhcoatl at LSVP UAMIztapalapa/DGTIC UNAM/CGSTIC CINVESTAV. M. Micoulaut acknowledges access to the supercomputer Roméo of Université de Reims Champagne-Ardennes.
1 A.D. LaLonde, Y. Pei, H. Wang, G.J. Snyder, Mat. Today 14, 526 (2011)

2 I. Galiuk, S.A. Miller, G.J. Snyder, O. Oeckler, Zeitschr. Anorg. Allg. Chem. 644, 1915 (2018).

3 C. Vigreux, M.V. Thi, G. Maulion, R. Kribich, M. Barillot, V. Kirschner, and Annie Pradel, Opt. Mater. Express 4, 1617 (2014).

${ }^{4}$ C. Vigreux, A. Piarristeguy, R. Escalier, S. Ménard, M. Barillot, and Annie Pradel, Phys. Status Solidi A 211, 932 (2011).

5 Amorphous Chalcogenides: Structure, Properties, Modeling and Applications, Ed. A. V. Kolobov, K. Shimakawa (World Scientific, Singapore, 2020)

6 Phase Change Materials : Science and Applications, S. Raoux, M. Wuttig Eds. (Springer, Berlin, 2009).

7 W. Wu, J. Mater. Sci. Mater. Electron. 27, 2183 (2016).

8 K.-H. Song S.-W. Kim, J-H. Seo, and H.-Y. Lee, Thin Sol. Films 517, 3958 (2009).

9 K. Wang, D. Wamwangi, S. Ziegler, C. Steimer, and M. Wuttig, J. Appl. Phys. 96, 5557 (2004).

10 E.M. Vinod, K. Ramesh, K.S. Sangunni, Sci. Rep. 5, 1 (2015).

11 C. Koch, A.-L. Hansen, T. Dankwort, G. Schienke, M. Paulsen, D. Meyer, M. Wimmer, M. Wuttig, L. K, and W. Bensch, RSC Adv. 7, 17164 (2017).

12 J. Tomforde, S. Buller, M. Ried, W. Bensch, D. Wamwangi, M. Heidelmann, and M. Wuttig, Solid State Sci. 11, 683 (2009).

13 X. Ji, L. Wu, M. Zhu, F. Rao, Z. Song, Z. Hu, S. Guo, L. Xu, X. Zhou, and S. Fenga, RSC Adv. 5, 24966 (2015).

14 L. Yang, J.Q.Li, R.Chen, Y.Li, F.S. Liu, and W.Q. Ao, J. Electron. Mater. 45, 5533 (2016).

15 R. Shekhawat, H. Pamuluri, V.E. Madhavan, and K. Ramesh, Sci. Rep. 11, 7604 (2021).

16 H.L. Kagdada, P.K. Jha, P. Śpiewak, and K.J. Kurzydłowski, Phys. Rev. B 97, 134105 (2018).

17 K. Jeong, S. Park, D. Park, M. Ahn, J. Han, W. Yang, H.-S. Jeong, and M.-H. Cho, Sci. Rep. 7, 955 (2017).

18 K.S Andrikopoulos, S.N. Yannopoulos, G.A. Voyiatzis, A.V. Kolobov, M. Ribes, and J. Tominaga, J. Phys. Condens. Matter 18, 965 (2006).

19 U. Argaman, R.E. Abutbul, E. Segev, and G. Makov, Cryst. Eng. Comm 19, 1 (2017).

20 J. Sun, X. Wang, Q. Nie, S. Dai, X. Zhang, B. Bureau, C. Boussard-Plédel, C. Conseil, J. Chinese Cera. Soc. 40, 1006 (2012).

21 R. Svoboda, D. Brandova, M. Chromcikova, M. Setnicka, J. Chovanec, A. Cerna, M. Liska, J. Malek, J. Alloys Comp. 695,
2434 (2017)

22 R. Svoboda, D. Brandova, J. Malek, J. Alloys Comp. 680, 427 (2016).

23 C. Vigreux, M. Vu Thi, G. Maulion, R. Kribich, M. Barillot, V. Kirschner, A. Pradel, Opt. Mater. Express 4, 1617 (2014).

${ }^{24}$ L. Guarneri, S. Jakobs, A. von Hoegen, S. Maier, M. Xu, M. Zhu, S. Wahl, C. Teichrib, Y. Zhou, O. Cojocaru-Mirédin, M. Raghuwanshi, C.-F. Schön, M. Drögeler, C. Stampfer, R. P. S. M. Lobo, A. Piarristeguy, A. Pradel, J.-Y. Raty, M. Wuttig, Adv. Mat., 2102356 (2021).

25 S.-X. Peng, Y. Cheng, J. Pries, S. Wei, H.-B. Yu1, M. Wuttig, Sci. Adv. 6, 1 (2020).

26 C.Gonçalves, R.Mereau, V. Nazabal, C. Boussard-Pledel, C. Roiland, E. Furet, M. Deschamps, B. Bureau, and M. Dussauze, J. Solid State Chem. 297, 122062 (2021).

27 L. Bouëssel du Bourg, C. Roiland, L. le Pollès, M. Deschamps, C. Boussard-Plédel, B. Bureau, C.J. Pickard, and E. Furet, Phys. Chem. Chem. Phys. 17, 29020 (2015).

28 A. Piarristeguy, M. Micoulaut, R. Escalier, P. Jovari, I. Kaban, J. van Eijk, J. Luckas, S. Ravindren, P. Boolchand, A.Pradel, J. Chem. Phys. 143, 074502 (2015).

29 M. Micoulaut, A. Piarristeguy, H. Flores-Ruiz, A. Pradel, Phys. Rev. B 96, 184204 (2017)

30 R. Car, and M. Marrinello, Phys. Rev. Lett. 55, 2471 (1985).

31 N.V. Ilawe, J.A. Zimmerman, and B.M. Wong, J. Chem. Theory Comput. 11 (2015) 5426.

32 G. Ferlat, M. Hellgren, F.-X. Coudert, H. Hay, F. Mauri, and M. Casula, Phys. Rev. Materials 3 (2019) 063603.

33 S. Grimme, J. Comput. Chem. 27, 1787 (2006).

34 J. P. Perdew, K. Burke, M. Ernzerhof, Phys. Rev. Lett. 77, 3865 (1996).

35 M. Micoulaut, M.-V. Coulet, A. Piarristeguy, M.R. Johnson, G. Cuello, C. Bichara, J.-Y. Raty, H. Flores-Ruiz, A. Pradel, Phys. Rev. B 89, 174205 (2014).

36 M. Micoulaut, J. Chem. Phys. 138, 061103 (2013)

37 M. Bauchy, A. Kachmar, M. Micoulaut, J. Chem. Phys. 141, 194506 (2014).

38 S. Chakraborty, P. Boolchand, M. Micoulaut, Phys. Rev. B 96, 094205 (2017).

39 M. Bauchy, M. Micoulaut, Nature Comm. 6, 6398 (2015).

40 B. Mantisi, M. Bauchy, M. Micoulaut, Phys. Rev. B 92, 134201 (2015).

41 O. Masson, pyTSRedX, a data reduction program to obtain the atomic pair distribution function (PDF) from X-ray total scatter- 
ing data, 2018

42 M. Micoulaut, J. Phys.: Cond. Matt. 31, 285402 (2019)

43 P.S. Salmon, and A. Zeidler, J. Stat. Phys. 114006 (2019).

44 C. Steimer, V. Coulet, W. Wełnic, H. Dieker, R. Detemple, C. Bichara, B. Beuneu, J. P. Gaspard, and M. Wuttig, Adv. Mater. 20, 4535 (2008).

45 J. Y. Raty, V. V. Godlevsky, J. P. Gaspard, C. Bichara, M. Bionducci, R. Bellissent, R. Céolin, J. R. Chelikowsky, and P.Ghosez, Phys. Rev. B 65, 115205 (2002)

46 J. Akola and R. O. Jones, Phys. Rev. Lett. 100, 205502 (2008).

47 J. M. Van Eijk, Structural analysis of phase change materialsusing x-ray absorption measurements, Ph.D. thesis, RWTH Aachen University, 2010.

48 D. A. Baker, M. A. Paesler, G. Lucovsky, S. C. Agarwal, andP. C. Taylor, Phys.Rev.Lett. 96, 255501 (2006).

49 J. R. Stellhorn, S. Hosokawa, W.-C. Pilgrim, N. Blanc, N.Boudet, H. Tajiri, and S. Kohara,Phys. Status Solidi 253, 1038 (2016).

50 P. Noé, C. Sabbione, N. Castellani, G. Veux, G. Navarro, V.Sousa, F. Hippert, and F. d'Acapito,J. Phys. D: Appl. Phys. 49, 035305 (2016).

51 M. Micoulaut, R. Vuilleumier, C. Massobrio, Phys. Rev. B 79 (2009) 214204.

52 A. Bouzid, G. Ori, M. Boero, E. Lampin, and C. Massobrio, Phys. Rev. B 96 (2017) 224204.

53 P.L. Silvestrelli, E. Martin, M. Boero, A. Bouzid, G. Ori, and C. Massobrio, J. Phys. Chem. B 124 (2020) 11273.

54 F. H. M. van Roon, C. Massobrio, E. de Wolff, and S. W. de Leeuw J. Chem. Phys. 113, 5425 (2000).

55 I. Petri, P.S. Salmon, and H.E. Fischer, J. Phys.: Condens. Matter 11, 7051 (1999).

56 H. Flores-Ruiz, M. Micoulaut, M.V. Coulet, A. Piarristeguy, M.R. Johnson, G.J. Cuello, A. Pradel, Physical Review B 92, 134205 (2015)

57 M. Micoulaut, K. Gunasekera, S. Ravindren, and P. Boolchand, Phys. Rev. B 90, 094207 (2014).

58 A.V. Marchenko, E.I. Terukov, F.S. Nasredinov, and P.P. Seregin, Tech. Phys. Lett. 46, 958 (2020).

59 J.-Y. Raty, W. Zhang, J. Luckas, C. Chen, R. Mazzarello, C. Bichara, and M. Wuttig, Nature Comm. 6, 7467 (2015).

60 G.B. Fisher, Proceedings of the NATO Advanced Study Institute on the Physics of Structurally Disordered Solids, S.S. Mitra Ed. (Springer, Berlin, 1976) p. 703.

61 J.-J. Kim, K. Kobayashi, E. Ikenaga, M. Kobata, S. Ueda, T. Matsunaga, K. Kifune, R. Kojima, and N. Yamada, Phys. Rev. B 76, 115124 (2007).

62 J. Akola, and R.O. Jones, Phys. rev. B 76, 235201 (2007).

63 A. Piarristeguy, A. Pradel, J.-Y. Raty, MRS Bull. 42, 45 (2017).

64 J. Luckas, A. Olk, P. Jost, H. Volker, J. Alvarez, A. Jaffré, P. Zalden, A. Piarristeguy, A. Pradel, C. Longeaud, M. Wuttig, Appl. Phys. Lett. 105, 092108 (2014).

65 A. Piarristeguy, M. Micoulaut, R. Escalier, G. Silly, M.-V. Coulet, A. Pradel, Journal of Non-Crystalline Solids 352, 120730 (2021).

66 G.B. Fisher and W. E. Spicer, J. Non-Cryst. Solids 8-10, 978 (1972)

67 N. J. Shevchik, J. Tejeda, D. W. Langer, M. Cardona, Phys. Stat. solidi B 21, 245 (1973).

68 C. Longeaud, J. Luckas, D. Krebs, R. Carius, J. Klomfass and M. Wuttig J. Appl. Phys. 112, 113714 (2012).

69 S. Wei, P. Lucas, and C.A.Angell, Mat. res. Bull. 44 (2019) 691.

70 A.L. Allred, J. Inorg. Nucl. Chem. 17 (1961) 215.

71 F. Kakinuma, S. Ohno, J. Phys. Soc. Jpn. 56 (1987) 619.

72 C. Persch, M.J. Müller, A. Yadav, J. Pries, N. Honné, P. Kerres, S. Wei, H. Tanaka, P. Fantini, E. Varesi, F. Pellizzer, and M. Wuttig, Nature Comm. 12 (2021) 4978.

73 W.A. Kamitakahara, R.L. Cappelletti, P. Boolchand, B. Halfpap, F. Gompf, D. A. Neumann, and H. Mutka, Phys. Rev. B 44, 94 (1991).

74 J.-Y. Raty, P. Noé, G. Ghezzi, S. Maîtrejean, C. Bichara, and F. Hippert, Phys. Rev. B 88, 014203 (2013).

75 G.C. Sosso, G. Miceli, S. Caravati, J. Behler, and M. Bernasconi, Phys. Rev. B 85, 174103 (2012).

76 R. Mazzarello, S. Caravati, S. Angioletti-Uberti, M. Bernasconi, and M. Parrinello, Phys. Rev. Lett. 104, 085503 (2010).

77 L. Guarneri, S. Jakobs, A. von Hoegen, S. Maier, M. Xu, M. Zhu, S. Wahl, C. Teichrib, Y. Zhou, O. Cojocaru-Mirédin, M. Raghuwanshi, C.-F. Schön, M. Drögeler, C. Stampfer, R.P.S.M. Lobo, A. Piarristeguy, A. Pradel, J.-Y. Raty, M. Wuttig, Adv. Mater. (2021) in press.

78 P.N. Sen, and M.F. Thorpe, Phys. Rev. B 15, 4030 (1977). 